\title{
ARID1A loss derepresses human endogenous retrovirus-H to modulate BRD4-driven transcription
}

\section{Kai Yuan ( $\nabla$ yuankai@csu.edu.cn )}

Institute of Molecular Precision Medicine https://orcid.org/0000-0001-7002-5703

\section{Chunhong Yu}

Institute of Molecular Precision Medicine, Xiangya Hospital, Central South University

Xiaoyun Lei

Institute of Molecular Precision Medicine, Xiangya Hospital, Central South University

\section{Fang Chen}

Institute of Molecular Precision Medicine, Xiangya Hospital, Central South University

\section{Song Mao}

Institute of Molecular Precision Medicine, Xiangya Hospital, Central South University

Lu Lv

Institute of Molecular Precision Medicine, Xiangya Hospital, Central South University

Honglu Liu

Institute of Molecular Precision Medicine, Xiangya Hospital, Central South University

\section{Xueying Hu}

Institute of Molecular Precision Medicine, Xiangya Hospital, Central South University

\section{Runhan Wang}

School of Life Sciences, Central South University

\section{Licong Shen}

Department of Gynecology, Xiangya Hospital, Central South University

\section{$\mathrm{Na}$ Zhang}

Institute of Molecular Precision Medicine, Xiangya Hospital, Central South University

\section{Yang Meng}

Center for Medical Genetics, School of Life Sciences, Central South University

\section{Yunfan Shen}

Center for Medical Genetics, School of Life Sciences, Central South University

\section{Pishun Li}

Institute of Molecular Precision Medicine, Xiangya Hospital, Central South University

\section{Shi Huang}

Central South University https://orcid.org/0000-0003-2674-2830

\section{Hao Shao}

Hunan Key Laboratory of Molecular Precision Medicine, Xiangya Hospital, Central South University 
Institute of Molecular Precision Medicine, Xiangya Hospital, Central South University

\section{Zhuohua Zhang}

Centrol South University

\section{Article}

Keywords: transposable elements, HERVH, colorectal cancer

Posted Date: August 6th, 2021

DOl: https://doi.org/10.21203/rs.3.rs-604974/v1

License: (c) (1) This work is licensed under a Creative Commons Attribution 4.0 International License. Read Full License

Version of Record: A version of this preprint was published at Nature Communications on June 17th, 2022. See the published version at https://doi.org/10.1038/s41467-022-31197-4. 


\section{Abstract}

The transposable elements (TEs) through evolutionary exaptation have become an integral part of human genome, offering ample regulatory sequences and shaping chromatin 3D architecture. While the functional impacts of TE-derived sequences on early embryogenesis are recognized, their role in malignancy has only started to emerge. Here we show that many TEs, especially the pluripotency-related endogenous retrovirus $\mathrm{H}(\mathrm{HERVH})$, are abnormally activated in colorectal cancer (CRC) samples. The transcriptional upregulation of HERVH is associated with mutations of several tumor suppressors including ARID1A. Knockout of ARID1A in CRC cells leads to increased accessibility at HERVH loci and enhanced transcription, which is dependent on ARID1B. Suppression of HERVH in CRC cells and patientderived organoids impairs tumor growth. Mechanistically, HERVH transcripts colocalize with nuclear BRD4 foci, modulate their dynamics, and co-regulate many target genes. Altogether, we uncover a critical role for ARID1A in restraining HERVH, which can promote tumorigenesis by stimulating BRD4-dependent transcription when ARID1A is mutated.

\section{Introduction}

We have been facing constant viral attacks during the course of evolution. While most viruses come and go, few have invaded and colonized the germline genome, becoming a significant fraction of transposable elements (TEs) that contribute more than $50 \%$ to the human nuclear DNA content ${ }^{1-4}$. Human TEs include DNA transposons, long terminal repeat (LTR) retrotransposons, and non-LTR retrotransposons. The majority of them has lost the ability to transpose during evolution and had long been regarded as functionless repetitive DNA. Recent studies however have begun to reveal that TEs are an abundant source of many regulatory sequences ${ }^{2,3,5,6}$, such as microRNAs (miRNAs) and long noncoding RNAs (IncRNAs) ${ }^{7-12}$, and that TEs are co-opted to serve important functions including transcriptional regulation, chromatin organization and 3D compartmentalization, especially during early embryogenesis and in embryonic stem cells (ESCs) ${ }^{6,13-23}$.

The endogenous retroviruses (ERVs), which have been identified half a century ago ${ }^{24}$, make up $8 \%$ of the human genome. They are LTR retrotransposons and have similar compositions to retroviruses, with internal coding sequences (gag-pro-pol-env) flanked by a pair of identical LTRs containing cis-regulatory elements for transcription. By estimation, there are 98,000 copies of ERVs and their derivatives, with human endogenous retrovirus $\mathrm{H}(\mathrm{HERVH})$ being one of the most abundant groups, comprising a total of $\sim 2000$ copies among which $\sim 100$ are close to full-length ${ }^{25,26}$. ERVs are largely in heterochromatin and transcriptionally repressed by an expanding battery of epigenetic mechanisms ${ }^{4,27,28}$, including methylation of histone $\mathrm{H} 3$ on lysine 9 (H3K9) or lysine 27 (H3K27), DNA methylation, as well as the RNA $\mathrm{N}(6)$-methyladenosine $(\mathrm{m}(6) \mathrm{A})$ modification ${ }^{29,30}$. Of note, these regulatory mechanisms are often redundant and function in a context-specific manner ${ }^{31,32}$, reflecting the sophisticated evolutionary arms race between viral sequences and the host genome $\mathrm{e}^{2-4}$. 
ERVs are not always inactive. During the profound epigenetic resetting in early embryonic development, ERVs are systematically transcribed in a stage-specific manner, coinciding with different cellular identities and differentiation potencies ${ }^{33}$. While a comprehensive understanding of ERVs function during early embryogenesis is yet to be established, recent studies have revealed the intimate relationship between HERVH and the human pluripotency network ${ }^{19}$. Depending on different variants of LTR (LTR7, LTR7Y, and LTR7A/B/C), the transcription of HERVH internal sequence (HERVH-int) is activated from 4-cell stage to blastocyst ${ }^{33}$. HERVH transcripts are also highly abundant in human ESCs as well as induced pluripotent stem cells (iPSCs), and moreover, the naïve-like pluripotency is associated with higher levels of HERVH expression ${ }^{15,16,19,26}$. Activation of HERVH promotes both the acquisition and maintenance of pluripotent states, by generating noncoding RNAs (ncRNAs) or producing chimeric transcripts with protein-coding genes via alternative splicing ${ }^{15,16}$. The transcriptionally active HERVH can also demarcate topologically associated domains (TADs) and help maintain a pluripotent chromatin architecture ${ }^{22}$. Cancer development in many aspects parallels the process of early embryogenesis. This includes regain the capacity of self-renewal and dramatic alterations in epigenetic landscapes. Interestingly, reactivation of HERVH is also observed in several types of human cancer, such as colorectal carcinomas (CRCs) ${ }^{34-39}$, however, a mechanistic insight into this reactivation is lacking and its functional consequence unclear.

The SWI/SNF (mating type SWItch/Sucrose NonFermentable) family chromatin remodelers, BAF, PBAF, and GBAF, regulate chromatin packing and transcription by controlling the dynamics of nucleosomes ${ }^{40}$. As a subunit of the BAF complex, ARID1A functions as a bona fide tumor suppressor and is mutated in approximately $8 \%$ of all human cancers ${ }^{40-44}$. Mutation of ARID1A sensitizes cancer cells to bromodomain and extraterminal domain (BET) inhibitors ${ }^{45,46}$, likely due to its indispensable role in maintaining normal enhancer function by influencing BRD4 activity ${ }^{42,46,47}$. How ARID1A mutation affects BRD4 remains unknown. Here, we show that loss of ARID1A results in an ARID1B-dependent upregulation of HERVH, whose transcripts partition into nuclear BRD4 foci and contribute to the BRD4-dependent gene regulatory network. This ARID1B-HERVH-BRD4 axis is crucial for the growth of CRC cells and patientderived organoid, offering novel treatment opportunities for ARID1A mutated cancers.

\section{Results}

HERVH is abnormally upregulated in CRCs

The majority of the human genome is comprised of various repetitive DNA sequences, most of which are transcribable. To globally characterize the expression of repetitive DNA elements in CRCs, we collected 521 colon adenocarcinoma (COAD) and 177 rectum adenocarcinoma (READ) RNA-seq data from The Cancer Genome Atlas (TCGA), filtered and grouped them according to the variables (Fig. S1A), and quantified the repeats expression using the human RepeatMasker Repeats annotation (https://genome.ucsc.edu/cgi-bin/hgTables). We first applied principal component analysis (PCA) to the gene expression as well as the repeats expression data from 51 normal and 631 tumor samples (Fig. $\mathrm{S} 1 \mathrm{~A})$. Both the genes and repeats showed distinct expression profiles that successfully demarcated the 
normal and tumor samples (Fig. 1A-1B and Supplementary Table 1). We next categorized the differentially expressed repeats. While the simple repeats were the most abundant, many LTR retrotransposons (also known as ERVs) showed altered expression between normal and tumor tissues (Fig. 1C and Supplementary Table 1). Of the 580 ERVs, 44 were downregulated and 84 upregulated in CRC tumor tissues (Fig. 1D and Supplementary Table 1). To validate these upregulated ERVs, we repeated the analysis with another independent RNA-seq dataset (GSE50760) of CRC tissues, and identified 17 ERVs that showed consistent upregulation. Specific activation of ERVs is linked with pluoripotency in embryonic cells $s^{6,20}$. We compared the upregulated ERVs in CRC tissues with that observed in early embryos and ESCs, and pinpointed two elements, HERVH-int and LTR7Y, that constitute a full-legth HERVH (Fig. 1E and Supplementary Table 1). Both elements showed increased expression in tumor tissues and their expressions were highly correlated with each other in the TCGA colorectal dataset (COREAD) (Fig. S1B-S1D and Supplementary Table 1). To further confirm the upregualtion of HERVH in CRCs, we performed RNAscope analysis for HERVH RNA on CRC tissue array. Compared with the matched peritumoral tissues, the tumoral tissues showed significantly stronger RNAscope signals (Fig. S1E-S1F). We then investigated the association of the expression levels of HERVH with the clinical outcomes of the CRC patients using the TCGA COREAD dataset, and observed that higher HERVH-int expression predicted poorer survival (Fig. 1F and Supplementary Table 1).

Molecular characterization of CRC samples have revealed 24 genes that are significantly mutated ${ }^{48}$. To interrogate the relationship between these gene mutations and the upregulation of HERVH, we selected 516 CRC samples with genetic variation data from the TCGA COREAD dataset, extracted their mutational signatures, and correlated the mutational status of one of the 24 genes with the expression of either HERVH-int or HERVK-int for comparison (Fig. S1A). In contrast to HERVK-int whose expression showed no obvious association with any gene mutations analyzed, the expression of HERVH-int correlated with the mutational status of several genes (Fig. $1 \mathrm{G}$ and Supplementary Table 1). We expanded this analysis to the 59 CRC cell lines in cancer cell line encyclopedia (CCLE) ${ }^{49}$ (Fig. $1 \mathrm{H}$ and Supplementary Table 1), and identified a list of genes whose mutation was consistently correlated with upregulation of HERVH. This included MLK4, FBXW7, ACVR1B, ARID1A, GRIK3, and SMAD2.

\section{Loss of ARID1A leads to transcriptional activation of HERVH}

Knockdown the expression of some of the listed genes by small interfering RNA (siRNA) resulted in increased transcription of HERVH (Fig. S1G). We selected ARID1A for further functional validation, because it is a DNA-binding subunit of the BAF chromosome remodeler complex and its inactivation mutations occur in a broad spectrum of human cancers ${ }^{40,41,43}$. 
To comprehensively depict the changes of repeats expression upon ARID1A loss, we collected and analyzed two independent RNA-seq data of HCT116 wild type (WT) and its isogenic ARID1A knockout (KO) cell lines ${ }^{42,50}$. Of note, the LTR retrotransposons or ERVs were the most upregulated repeat group in ARID1A KO cells (Fig. 2A, S2A, and Supplementary Table 2). We ranked all the ERVs according to their fold changes (Fig. 2B, S2A, and Supplementary Table 2). HERVH-int and two of its associated LTRs, LTR7Y and LTR7, were the three most significantly upregulated elements, whereas other HERVH-related LTRs didn't show consistent upregulation (Fig. 2C, S2A, and Supplementary Table 2). Scatter plots of the expression of all 580 ERVs revealed that HERVH-int, LTR7Y, and LTR7 were already expressed in HCT116 WT cells but the ARID1A inactivation further increased their abundance (Fig. 2D, S2C, and Supplementary Table 2). These observations were further validated with our own RNA-seq data of ARID1A WT and KO HCT116 cells (Fig. S2B, S2D, and Supplementary Table 2). Overlapping the significantly upregulated ERVs in the three datasets spotted HERVH-int and its LTR as the only unambiguously activated elements upon ARID1A loss (Fig. 2E). We generated additional ARID1A KO colorectal cell lines to further confirm the observed upregulation of HERVH (Fig. 2F). qPCR analyses with primers specifically targeting the gag and $p o /$ sequences of HERVH-int revealed increased transcripts abundance in all three ARID1A KO cell lines (Fig. $2 \mathrm{G}$ and $2 \mathrm{H}$ ). To test if the transcriptional activation of HERVH can be suppressed by reexpression of ARID1A, we infected the ARID1A KO cells with lentiviruses carrying ARID1A or its counterpart ARID1B ${ }^{51,52}$ (Fig. 2I-2K). The re-introduction of ARID1A significantly downregulated the expression of HERVH. Interestingly, overexpression of ARID1B didn't rescue but instead mildly increased the amount of HERVH transcripts in ARID1A KO cells (Fig. 2K).

Unlike genes, ERVs are quite diverse between primates and rodents. To assess the effect of ARID1A inactivation on ERVs expression in mice, we analyzed RNA-seq data of the colon epithelial cells from WT or ARID1A KO mice ${ }^{42}$. Of the 423 ERVs in the mouse genome, 16 were downregulated and 11 upregulated in the absence of ARID1A (Fig. S2E and Supplementary Table 3). The upregulated ERVs included RLTR1Bint, RLTR1D, RMER12B, IAPLTR4_I (Fig. S2F and Supplementary Table 3). Therefore, the influence of ARID1A on ERVs seemed to be universal, and in human, the most responsive element toward ARID1A mutation was the HERVH.

\section{Transcription of HERVH in the absence of ARID1A is dependent on ARID1B}

To investigate the mechanism of how ARID1A loss induced HERVH transcription, we put our focus on ARID1B, which shares $60 \%$ homology with ARID1 $A^{51}$. Functioning as the rigid structural core, they are mutually exclusive in the BAF complex ${ }^{52,53}$. ARID1B is essential for the survival of ARID1A mutated cancer cells, by supplying residual BAF complex activities to maintain chromatin accessibility at enhancers and regulate RNA polymerase II dynamics $50,54,55$. 
We first examined the influence of ARID1B on the expression of repetitive elements using published RNAseq data ${ }^{50}$ (Fig. 3A and Supplementary Table 4). Knocking down the expression of ARID1B by short hairpin RNA (shRNA) in WT HCT116 cells (WT-KD) only showed limited effects on repeats expression, however, ARID1B knockdown in ARID1A KO cells (KO-KD) dramatically reduced the transcripts abundance of many repeats, especially the LTR retrotransposons (ERVs) (Fig. 3B and Supplementary Table 4). Of note, the upregulation of several HERVH elements was partially reversed by ARID1B KD (Fig. 3C and Supplementary Table 4). Using two different shRNAs targeting ARID1B, we verified the suppression of HERVH by ARID1B KD in ARID1A KO cells (Fig. 3D). Both ARID1A and ARID1B harbor DNA-binding activity ${ }^{51}$. We next analyzed their occupancy on the HERVH elements using ChIP-qPCR (Fig. S3A and S3B). While the amount of ARID1A on HERVH was minimized in ARID1A KO cells, the binding of ARID1B to HERVH was compensatorily increased, maintaining a comparable amount of BAF activity at HERVH loci in these cells (Fig. S3C). The ARID1A- and ARID1B-containing BAF complexes are associated with different histone acetyltransferase (HAT) and deacetylase (HDAC) activities ${ }^{52}$. To characterize the epigenetic changes accompanying this subunit switch of BAF complex on HERVH, we identified the commonly derepressed genomic HERVH loci in different datasets and analyzed their chromosome accessibility as well as histone modifications (Fig. S3D and Supplementary Table 5). In ARID1A KO HCT116 cells, we observed some increase in accessibility at the HERVH loci (Fig. 3E, 3H, and Supplementary Table 5). Interestingly, acetylation of H3K27 (H3K27ac), as well as mono methylation of histone 3 on lysine 4 (H3K4me), was also increased (Fig. 3F-3H, and Supplementary Table 5). We further validated this increase of H3K27ac on HERVH by ChIP-qPCR (Fig. S3E). To test whether HDACs and HATs contributed to the dysregulation of HERVH in the absence of ARID1A, we treated WT HCT116 cells with two different HDAC inhibitors, SAHA and TSA. Both inhibitors stimulated the expression of HERVH (Fig. S3F). We treated the ARID1A KO cells with MG149, an inhibitor of Tip60 which is a HAT associated with ARID1B-BAF complex ${ }^{52}$. The inhibition of Tip60 suppressed the activation of HERVH upon ARID1A loss (Fig. S3G). To identify which transcription factor (TF) accounted for the increased expression of HERVH, we examined the expression levels of all the TFs that are able to bind HERVH in ARID1A KO HCT116 cells (Fig. S3H), individually knocked down their expression by siRNA, and performed RNA-seq analysis (Fig. S3I and Supplementary Table 6). Only SP1 knockdown significantly reduced the expression of HERVH (Fig. S3J).

Based on these results, we propose that the ARID1A-contaning BAF complex normally maintains a compact chromatin configuration at HERVH loci with the help from its associated HDACs. When ARID1A is mutated, the ARID1B-containing BAF recruits HATs to the HERVH loci and increases local accessibility, and then SP1 binds to and activates its transcription (Fig. 3I). 
Knockdown the expression of HERVH in ESCs triggers differentiation ${ }^{15}$. To investigate the function of HERVH transcription in CRCs, we reduced its expression in different cell lines and patient-derived organoids using shRNAs targeting different regions of HERVH-int and assessed the consequence (Fig. S4A).

We first tested the effects of HERVH knockdown with different colorectal cell lines. The cell lines analyzed all had varying degrees of HERVH expression, whereas the expression of HERVK was kept to a minimum (Fig. S4B). Cell viability assay showed that knockdown of HERVH impaired the survival of all the cell lines tested (Fig. S4C), and the HERVH knockdown also strongly inhibited colony formation of these cells in clonogenic assays (Fig. S4D). CRC cell line SW480 had weak ability in the formation of tumor spheres when cultured in ultra-low attachment plates, and ARID1A inactivation significantly enhanced this ability (Fig. 4A). Knockdown of HERVH in the ARID1A KO cells greatly reduced the formation of tumor spheres (Fig. 4A-4C). To further assess the function of HERVH in tumorigenicity, we subcutaneously seeded shRNA-infected ARID1A WT or KO HCT116 cells into nude mice. The cells with HERVH knockdown showed significant growth impairment when compared with control cells (Fig. 4D).

We next sought to verify the critical role of HERVH in patient-derived CRC organoids. We obtained tumoral and peritumoral tissues from surgical biopsy, and evaluated their HERVH transcripts level using qPCR (Fig. S4E). The tumoral tissues with high HERVH expression were selected to generate CRC organoids, which were then infected with lentiviruses carrying shRNA targeting either HERVH or GFP to achieve specific knockdown (Fig. 4E). RNA fluorescence in situ hybridization (FISH) of the organoids confirmed the knockdown efficacy of HERVH (Fig. S4F). Compared to control, HERVH knockdown resulted in the formation of fewer organoids (Fig. 4F), and their size was also much smaller (Fig. S4G, S4H, and 4G). We examined the cell proliferation and apoptosis in the treated organoids by Ki67 and TUNEL stainings (Fig. $4 \mathrm{H}$ and $4 \mathrm{I}$ ). HERVH knockdown dramatically reduced the number of proliferating Ki67 positive cells (Fig. $4 \mathrm{~J}$ ), meanwhile increased the number of TUNEL positive apoptotic cells (Fig. 4K).

Altogether, the results suggested that HERVH is a vulnerability not only in ARID1A mutated cells, but also in many other CRC cells that express HERVH. 
HERVH is part of the transcriptional circuitry regulating pluripotency, and its transcription markedly influences the transcriptome ${ }^{13,15,16,56}$. To investigate the molecular underpinnings of the oncogenic function of HERVH in CRCs, we assessed the impact of HERVH knockdown on global gene expression. PCA showed that the transcriptomes of the ARID1A WT and KO HCT116 cells were noticeably separated on the second principal component (PC2), and HERVH knockdown narrowed this difference (Fig. 5A), suggesting that the altered transcription seen in ARID1A KO cells was partially linked to the upregulation of HERVH. We identified 552 upregulated genes and 531 downregulated genes whose transcriptional change was reversed upon HERVH knockdown in ARID1A KO cells (Fig. 5B and Supplementary Table 7). Many of the $552 \mathrm{HERVH}$-dependent upregulated genes were enriched in cancer related pathways (Fig. 5C and Supplementary Table 7). We selected some of the upregulated target genes and validated the observed reversion of expression by qPCR (Fig. S5A-S5B). The observed increase of H3K27ac and H3K4me at the derepressed HERVH loci suggested that they could function as active enhancers and their transcripts enhancer RNAs ${ }^{57}$ (Fig. $3 F$ and $3 G$ ). Additionally, HERVH transcripts are able to interact with many subunits of the mediator complex ${ }^{15}$. We compared the transcriptome dynamics after siRNA mediated knockdown of different subunits of the mediator complex, its binding partner BRD4, and HERVH. The correlation matrix suggested that suppression of BRD4 and HERVH imposed similar influence on global transcription (Fig. S5C and Supplementary Table 7). Further analysis using the differentially expressed genes in BRD4 and HERVH knockdown cells revealed strong correlation between these two groups (Fig. 5D, S5D, and Supplementary Table 7). Of the 1643 differentially expressed genes upon HERVH knockdown, 1018 of them showed similar changes in BRD4 knockdown cells (Fig. 5E and Supplementary Table 7).

BRD4 as well as the mediator complex subunit MED1 can form liquid-like condensates, especially at super-enhancers ${ }^{58,59}$. To investigate the distribution of HERVH transcripts and their relationship with BRD4 and the mediator complex, we combined RNA FISH targeting HERVH with immunofluorescence (IF) stainings. The specificity of the RNA FISH was validated by the absence of signals in HERVH knockdown cells (Fig. S5E). Varying degrees of colocalization was detected between HERVH transcripts and the endogenous BRD4, MED1, and MED12 (Fig. S5F), suggesting that HERVH RNA could regulate their protein dynamics in the nucleus. We knocked down the expression of HERVH, and observed a mild decrease in the protein level of BRD4 (Fig. 5G). To further characterize the influence of HERVH on BRD4, we stably expressed GFP-BRD4 in ARID1A KO HCT116 cells. GFP-BRD4 formed nuclear condensates as previously reported ${ }^{58}$, and RNA FISH revealed clear distribution of HERVH RNA in the BRD4 puncta (Fig. 5F). Knockdown of HERVH markedly decreased both the number and size of the BRD4 puncta (Fig. $5 \mathrm{H}$ and 5I). We further assessed the dynamics of the BRD4 puncta using fluorescence recovery after photobleaching (FRAP). While in control cells the photobleached BRD4 puncta quickly recovered its fluorescence, the fluorescence recovery after HERVH knockdown became much slower (Fig. 5J). 
It was reported that ARID1A mutant cells showed higher sensitivity to the BET inhibitor JQ1 ${ }^{45,46}$. We confirmed that the ARID1A KO HCT116 cells were indeed more sensitive to JQ1 as well as another recently reported BRD4 inhibitor NHWD-870 ${ }^{60}$ (Fig. S5G and S5H). The results reported here suggested that the upregulated transcription of HERVH in ARID1A mutant cells contributed to the formation of BRD4 nuclear puncta and stimulated their dynamic activity (Fig. 5K), providing an explanation for the observed increased sensitivity to BET inhibitors.

\section{Discussion}

Mutational landscape analyses have revealed that ARID1A is among the most frequently mutated epigenetic factors across many cancer types ${ }^{40,44}$. Understanding its mechanism of action and hence identifying targetable vulnerabilities for ARID1A inactivation have been of great importance. In this study, we investigated how the repetitive genome responded to the inactivation of ARID1A and identified that the HERVH group of ERVs was specifically derepressed. This derepression was ARID1B-dependent, and was indispensable for the survival of the CRC cells, likely due to its influence on the dynamics of BRD4 and the regulated transcriptional network. Several synthetic lethality targets of ARID1A have been reported, including ARID1B, EZH2, HDAC6, Aurora A, and GCLC 50,54,61-66. ARID1A mutant cells are also hypersensitive to BET inhibitors ${ }^{45,46}$, a promising class of anticancer drugs. Our results suggest that the activation of pluripotency-related HERVH is a shared mechanistic foundation of the previously observed ARID1B- and BET-vulnerabilities of the ARID1A mutated tumors. The ARID1B-HERVH-BRD4 regulatory axis and the adjunct mechanism reported here also offer several new potential targets of intervention (Fig. 5K), among which the HERVH itself is of most interest, because of its specific expression in early embryos and general silencing in most adult tissues. It is worth noting that ARID1A has been implicated in several other biological processes, some of which also involves HERVH, such as high-order spatial chromosome partitioning and tissue regeneration ${ }^{15,16,22,67-69}$. The molecular mechanism reported here may have certain explanatory power in those scenarios as well.

Derived from ancient retroviral infections, ERVs are domesticated viral fossils in our genome whose activity is under close surveillance ${ }^{1,2,4,28}$. Comprehensive interrogations in mouse ESCs have revealed that overlapping epigenetic pathways linked to heterochromatin formation are enlisted to suppress the transcription of ERVs. This includes DNA methylation (5-methylcytosine, 5mC), various histone modifications (H3K9me3, H3K27me3, H4K20me3, H4R3me2, and H2AK119ub), and their corresponding writers and readers ${ }^{31,32}$. Reminiscent of the diversity of the process of heterochromatin formation in early embryos $^{70}$, different families of ERVs rely on distinct epigenetic means to achieve silencing. The specific recognition of different ERVs by the various epigenetic mechanisms is in part mediated by the KRAB domain-containing zinc finger proteins (KZFPs), which can bind to specific DNA sequences in individual ERV and recruit KAP1 and other epigenetic modifiers. RNA mediated targeting mechanisms also contribute to the specific silencing of ERVs. piRNAs as well as other small RNA species are able to bring histone modifying activities to their complementary ERV loci ${ }^{4,28,71,72}$. Our study reveals another mode of ERVs suppression which involves the SWI/SNF chromatin remodelers, further increasing the complexity 
of the epigenetic regulatory network constraining the expression of ERVs. The targeting mechanism for the BAF complex in silencing HERVH is currently unknown. It will be interesting to investigate the potential interactions between BAF and KZFPs or the small RNA machineries.

Accumulating evidence reveals that ERVs are co-opted to perform a wide range of biological functions. In early embryos and ESCs, ERVs serve as regulatory elements and alternative promoters to rewire the transcription network of pluripotency ${ }^{13,21}$. Moreover, certain groups of ERVs become transcriptionally activated in an orderly fashion during embryogenesis ${ }^{33}$, functioning as enhancer or long noncoding RNAs $^{14,15}$, and sometimes synthesizing reverse transcriptase activity and even forming viral-like particles $^{73}$. ERVs are also involved in many human diseases such as various types of cancer. The abnormally activated ERVs can produce long noncoding RNAs or functional polypeptides $37,74-77$, enabling cancer cells to exploit and repurpose developmental pathways to promote malignancy ${ }^{38}$. Of particular note, the reactivated ERVs in cancer are extensively recruited as promoters to drive expression of many oncogenes in a process termed onco-exaptation ${ }^{78,79}$. Our results reciprocally demonstrate that mutations of tumor suppressor can activate functionally important ERVs, suggesting the existence of positive feedback loops between ERVs and cancer driver genes. Future studies shall extend the analysis to other cancer driver genes and characterize these positive feedback loops more comprehensively. The establishment of a mutually reinforcing relationship between cancer driver genes and ERVs will deepen our understandings on the etiology of malignancy and throw new light on cancer treatments.

\section{Methods}

Data download

The TCGA dataset used in this study, including the RNA-seq BAM files, the gene raw count data (htseqcount files), and the annotated somatic simple nucleotide variation files (MuTect2 VCF) of patients with colon adenocarcinoma (COAD) and rectum adenocarcinoma (READ), were accessed through dbGaP accession number phs000178.v11.p848 and downloaded using the gdc-client v1.6.0. The cinical overall survival (OS) information was obtained from Liu et al.80. The RNA-seq fastq files of normal and tumor tissues from another $18 \mathrm{CRC}$ patients were downloaded from https://www.ncbi.nlm.nih.gov/geo under the accession number GSE5076081. The RNA-seq fastq files of the 59 colorectal cancer cell lines in cancer cell line encyclopedia (CCLE) were downloaded from https://www.ebi.ac.uk/ under the accession number PRJNA52338049, and the corresponding germline filtered CCLE merged mutation calls were acquired from https://portals.broadinstitute.org/ccle/data. The previously published RNA-seq and ChIPseq raw reads fastq files generated with HCT116 cells or mice primary colon epithelial cells were downloaded from https://www.ncbi.nlm.nih.gov/geo under the accession numbers GSE71514 and GSE10196642,50.

RNA-seq analysis 
Raw reads were first cleaned using trim_galore v0.6.0 (http://www.bioinformatics.babraham.ac.uk/projects/trim_galore/) with default parameters. The reads from each RNA-seq sample were then mapped to hg38 or mm9 genome assembly downloaded from UCSC, using STAR v2.5.3a82. The key alignment parameters were as follows: "--

outFilterMismatchNoverLmax 0.04 -outSAMtype BAM SortedByCoordinate -outFilterMultimapNmax 500 -outMultimapperOrder Random --outSAMmultNmax 1"; the parameters "-outFilterMultimapNmax 500" and "--outMultimappedOrder Random" ensured that multiple aligned reads were included but only one position was assigned randomly. Genes expression was quantified using featureCounts v1.6.583 of subread-1.6.5 package based on hg38 RefSeq genes annotation file. Repeats expression was quantified using featureCounts v1.6.5 ("featureCounts --M -fraction") based on repeats annotation files downloaded from https://genome.ucsc.edu/cgi-bin/hgTables. Principal component analysis was conducted with the functions "vst" and "plotPCA" from R package DESeq2 v1.22.284. Differential expression analysis was performed based on the negative binomial distribution using the functions "DESeq" and "results" from DESeq2. The heatmap of differentially expressed genes or repeats was created using $\mathrm{R}$ package pheatmap v1.0.12. The KEGG enrichment analysis was performed using the function "enrichKEGG" from the R package clusterProfiler v3.10.185. Venn diagrams were prepared with the R package Vennerable and venn.

Survival analysis

The curated clinical endpoint results (OS event and OS event times) of the 628 patients in TCGA-COREAD dataset were obtained from Liu et al.80. Only patients in stages II and later according to the American Joint Committee on Cancer (AJCC) pathologic tumor staging system were included. The 493 CRC patients were classified into HERVH-high (145 patients with HERVH-int CPM>8430.797) and HERVH-low groups (348 patients with CPM<8430.797), and the survival curves of the two groups were compared using log-rank test from the function "survdiff" in R package survival v2.44-1.1.

Integration analysis of whole-exome sequencing (WXS) and RNA-seq

WXS files (MuTect2 VCF) and RNA-seq data from 516 patients in TCGA-COREAD were analyzed (Fig. $\mathrm{S} 1 \mathrm{~A})$. All the somatic mutational information was included regardless of their classification. For each gene, we classified the patients into WT or mutation group, and then calculated the Log2 FoldChange between these two groups using the expression values (CPM) of HERVK-int and HERVH-int. p values were calculated by Wilcoxon test.

ATAC-seq and ChIP-seq analyses

Page $12 / 33$ 
Raw reads were cleaned using trim_galore. The reads were then aligned to the hg38 genome assembly using Bowtie2 v2.3.5.186, with the default parameters that look for multiple alignments but only report the one with best mapping quality. Duplicate reads were then removed using MarkDuplicates from gatk package v.4.1.4.1. Replicate samples were merged using the samtools v1.1087. Peak calling was performed using MACS2 v2.2.688 (parameters: -g hs -keep-dup 1 -broad-cutoff 0.01). Peaks near active HERVH loci were identified using bedtools v2.26.089. For ATAC-seq, bigwig tracks were generated using bamCoverage from python package deeptools (parameteres: --skipNAs -normalizeUsing CPM) 90 . For ChIP-seq, bigwig tracks were generate using bamCompare from deeptools (parameters: --skipNAs scaleFactorsMethod readCount --operation log2 --extendReads 200). Negative values were set to zero. ATAC-seq and ChIP-seq profiles were created by computeMatrix and plotProfile in deeptools. IGV v.2.4.13 was used to visualize the bigwig tracks91.

Cell culture and cell line generation.

The cell lines used in this study, including HCT116, DLD1, SW480, LS174T, SW620, HT29, HCT8, RKO, CRL1790/841, NCM460, and 293T, were cultured in RPMI 1640 or DMEM medium containing 10\% FBS and incubated at $37^{\circ} \mathrm{C}$ with $5 \% \mathrm{CO} 2$ in a humidified incubator. To generate ARID1A KO cell lines, the indicated cells were transfected with LentiCRISPR-V2 plasmid carrying sgARID1A (Supplementary Table 8) using Lipofectamine 2000 (Invitrogen), and further selected by $1 \mathrm{mg} / \mathrm{mL}$ puromycin (Selleck, s7417) for 3 days. The cells were then plated at single-cell density in $100 \mathrm{~mm}$ petri dishes, and the emerged individual clones were picked and replated into 24-well plates. The loss of ARID1A expression was confirmed by western blot.

Organoid culture

The CRC organoid was generated as previously described92. All the human tissue related experiments were approved by the Medical Ethics Committee of Central South University, and the informed consent was obtained from the patients. From the resected colon segment, the tumor tissues as well as normal tissues were isolated and stored in ice-cold RPMI 1640 supplemented with 1\% Penicillin-Streptomycin. The tissues were then washed in ice-cold DPBS supplemented with $1 \%$ Penicillin-Streptomycin and cut into 1-3 $\mathrm{mm} 3$ cubes. After centrifuging at $200 \mathrm{~g}$ for $5 \mathrm{~min}$, the supernatant was removed and pellet was resuspended in collagenase IV (Gibco, 17104019) supplemented with $10 \mu \mathrm{M}$ ROCK inhibitor Y-27632 dihydrochloride (Merk Millipore, SCM075). The tissues were digested at $37^{\circ} \mathrm{C}$ for 1 hour and mixed up every 10-15 min by pipetting, washed with $10 \mathrm{~mL}$ advanced DMEM/F12 (Thermo Fisher Scientific, 12634010) supplemented with $\mathrm{Y}-27632$, and then centrifuged at $200 \mathrm{~g}$ for $5 \mathrm{~min}$ at $4{ }^{\circ} \mathrm{C}$. The pellet was resuspended in DMEM/F12 supplemented with Y-27632 and filtered through $60 \mu \mathrm{m}$ cell strainer. After centrifugation at $200 \mathrm{~g}$ for $5 \mathrm{~min}$ at $4{ }^{\circ} \mathrm{C}$, the supernatant was discarded and the pellet resuspended in $70 \%$ Matrigel (Corning, 356231). $30 \mu \mathrm{L}$ of the Matrigel mixture was plated on the bottom of 24-well plates, 
and $500 \mu \mathrm{L}$ organoid medium (Accurate International Biotechnology, M102-50) was added to each well following incubation at $37^{\circ} \mathrm{C}$ with $5 \% \mathrm{CO} 2$ for 30 min. The organoid medium was changed every $2-3$ days, and the organoids were passaged after 7 days of culture.

Cell growth assays

For cell viability assays, cells were plated into 96-well plates at the density of 2000-5000 cells per well after infected with lentiviruses expressing shGFP or shERVH. The cells were kept for another 7 days, and the viability was measured daily using MTT (Sigma, M5655) as previously described 93 . For chemosensitivity assays, the cells were seeded in 96-well plates and treated with the compounds at indicated concentrations for 72 hours, and then the cell viability was measured. For colony formation assays, the cells were seeded at the density of 1000-2000 cells per well in 6-well plates after infected with lentiviruses expressing shGFP or shERVH. The cells were allowed to grow for 10-14 days and then fixed for $10 \mathrm{~min}$ in $50 \%(\mathrm{v} / \mathrm{v})$ methanol containing $0.01 \%(\mathrm{w} / \mathrm{v})$ crystal violet.

\section{Tumor sphere formation}

The 6-well plates were coated with $12 \mathrm{mg} / \mathrm{mL}$ poly-hydroxyethylmethacrylate (polyHEMA, Sigma-Aldrich, P3932) in 95\% ethanol. The indicated cells were digested by TrypLE, and approximately 1000 cells were suspended in 50\% Matrigel (Corning, 356231) and plated in the precoated 6-well plates. The 6-well plates containing the cells were incubated at $37^{\circ} \mathrm{C}$ for $30 \mathrm{~min}$, and then $2 \mathrm{~mL}$ of phenol red-free DMEM/F12 (GIBCO, 21041) containing 1× B27 supplement (Invitrogen, 12587) and $20 \mathrm{ng} / \mathrm{mL}$ rEGF (Sigma Aldrich, E9644) was added into each well. The culture medium was changed every 2-3 days, and the number of tumor spheres in each well was counted after 12 days.

\section{Xenograft tumors}

The 4-5 weeks old female BALB/c nude mice were purchased from Hunan SJA Laboratory Animal Co., Ltd. (Changsha, China). $5 \times 105$ of the indicated cells were suspended in $100 \mu \mathrm{L}$ DPBS and injected subcutaneously into the flank of nude mice. The tumors were measured twice weekly with an electronic caliper, and the volumes were calculated using the formula: $0.5 \times$ (length $\times$ width2). All the animal experiments were approved by the Medical Ethics Committee of Central South University, and conducted according to the Guidelines of Animal Handling and Care in Medical Research in Hunan Province, China. 
The siRNA oligos were synthesized by GenePharma (Shanghai GenePharma Co., Ltd.), and the sequences were listed in Supplementary Table 8. Cells were transfected with the indicated siRNA by Lipofectamine 2000. After 48 hours, the cells were harvested and the efficiency of silencing was verified by qPCR. For shRNA, shRNA oligos were synthesized by Tsingke (Tsingke Biotechnology Co., Ltd.) and cloned into pLKO.1 TRC Cloning vector (Supplementary Table 8). The shRNA and packaging vectors (pMD2.G and psPAX2) were transiently co-transfected into 293T cells by polyethylenimine (Sigma, P3143), and the resulted lentivirus particles were harvested and precipitated by PEG8000. The target cells were treated with lentivirus particles and $8 \mu \mathrm{g} / \mathrm{mL}$ polybrene for 24 hours, and the efficacy of shRNA interference was determined by qPCR.

HERVH knockdown in organoids

The organoids cultured in Matrigel were washed once with DPBS, and digested with TrypLE for 5 min at $37^{\circ} \mathrm{C}$. During the digestion, Matrigel was disrupted by pipetting repeatedly. When cell clumps containing 2-10 cells were observed, $10 \mathrm{~mL}$ of advanced DMEM/F12 was added before centrifugation at $200 \mathrm{~g}$ for 5 min. The supernatant was removed and the cells were resuspended using organoid medium supplemented with $8 \mu \mathrm{g} / \mathrm{mL}$ polybrene. Then the cells were split equally into 2 wells of 24-well plate precoated with polyHEMA, and $50 \mu \mathrm{L}$ of lentivirus carrying shGFP or shERVH was added. After spin infection at $2000 \mathrm{rpm}$ for 1 hour, the cells were incubated at $37^{\circ} \mathrm{C}$ with $5 \% \mathrm{CO} 2$ for 4 hours. The cells were then resuspended in $10 \mathrm{~mL}$ of advanced DMEM/F12 and centrifuged at $200 \mathrm{~g}$ for $5 \mathrm{~min}$. The pellet was resuspended with $100 \mu \mathrm{L}$ of $70 \%$ Matrigel, and $10 \mu \mathrm{L}$ of the mixture was plated per well into prewarmed 96-well plate. The organoids were cultured for 10-14 days and the medium was changed every 2-3 days.

\section{Western blot}

Cells were washed with cold DPBS for two times and then lysed in $2 \times$ Laemmli buffer (2\% SDS, $20 \%$ glycerol, and $125 \mathrm{mM}$ Tris-HCl, $\mathrm{pH}$ 6.8) supplemented with $1 \times$ protease inhibitor cocktail (Sigma, P8340). The cell lysate was scraped and sonicated, and the concentration of protein was determined by BCA assay. The protein was separated by SDS-PAGE and transferred onto nitrocellulose membrane. The membrane was then blocked with $5 \%$ non-fat milk for 1 hour at room temperature, and incubated with the indicated primary antibody overnight at $4{ }^{\circ} \mathrm{C}$ with shaking. The membrane was washed for 3 times and incubated with secondary antibodies (1:5000, Thermo Fisher Scientific) for 2 hours. The signal was then detected with ECL substrates (Millipore, WBKLS0500). Dilutions of primary antibodies were: rabbit antiARID1A/BAF250A Ab (1:1000, Cell Signaling, 12354S), rabbit anti-BRD4 Ab (1:1000, Active Motif, 39909), mouse anti-a-Tubulin Ab (1:3000, Cell Signaling, 3873s). Primary antibodies used in this study were listed in Supplementary Table 11. 
RNA-seq and qPCR

The RNA of the treated cells was extracted by TRIzol (Life Technologies, 87804) according to the manufacturer's protocol. Total RNA was made into libraries for sequencing using the mRNA-Seq Sample Preparation Kit (Illumina) and sequenced on an Illumina Hiseq platform (Novagene, Tianjin, China). The sequencing data was deposited to the GEO database (accession number GSE). For RT-qPCR, RNA was extracted by TRIzol, and reverse transcribed to CDNA using the PrimeScript RT reagent Kit (Takara, RR037A). The CDNA was then used as templates and qPCR was performed using the SYBR Green qPCR Master Mix (SolomonBio, QST-100) on the QuantStudio 3 Real-Time PCR system (Applied Biosystems). Primers used in qPCR were listed in Supplementary Table 8.

\section{Chromatin immunoprecipitation}

The indicated cells in $100 \mathrm{~mm}$ petri dishes were cross-linked with $1 \%$ formaldehyde for $10 \mathrm{~min}$ at room temperature, and quenched with $125 \mathrm{mM}$ ice-cold glycine. The cells were then rinsed with $5 \mathrm{~mL}$ ice-cold $1 \times$ PBS for two times, and harvested by scraping using silicon scraper. After spinning at $1350 \mathrm{~g}$ for $5 \mathrm{~min}$ at $4^{\circ} \mathrm{C}$, the supernatant was discarded, and the pellet was resuspended in Lysis Buffer I ( $50 \mathrm{mM} \mathrm{HEPES}$ $\mathrm{KOH}, \mathrm{pH} 7.5,140 \mathrm{mM} \mathrm{NaCl}, 1 \mathrm{mM}$ EDTA, $10 \%$ glycerol, 0.5\% NP-40, 0.25\% Triton X-100 and $1 \times$ protease inhibitors) and incubated at $4{ }^{\circ} \mathrm{C}$ for 10 min with rotating. After spinning at $1350 \mathrm{~g}$ for $5 \mathrm{~min}$ at $4{ }^{\circ} \mathrm{C}$, the pellet was resuspended in Lysis Buffer II (10 mM Tris-HCl, pH 8.0, 200 mM NaCl, 1 mM EDTA, 0.5 mM EGTA and $1 \times$ protease inhibitors), incubated for $10 \mathrm{~min}$ at room temperature, and spun at $1350 \mathrm{~g}$ for 5 min at $4^{\circ} \mathrm{C}$. The pellet was again resuspended in Lysis Buffer III (10 mM Tris-HCl pH 8.0, $100 \mathrm{mM} \mathrm{NaCl}, 1$ mM EDTA, 0.5 mM EGTA, 0.1\% Na-Deoxycholate, 0.5\% N-lauroylsarcosine and 1× protease inhibitors) and transferred into Covaris microTUBEs. The DNA was sonicated to $200 \mathrm{bp}$ fragments using Covaris S220 (duty cycle: 10 ; intensity: 4; cycles/burst: 200; duration: 200 s). After quenching the SDS by $1 \%$ of Triton $\mathrm{X}-100$, the lysate was spun at $20,000 \mathrm{~g}$ for $10 \mathrm{~min}$ at $4^{\circ} \mathrm{C} .50 \mu \mathrm{L}$ of supernatant from each sample was reserved as input, and the rest lysate was incubated overnight at $4^{\circ} \mathrm{C}$ with the magnetic beads bound with ARID1A (CST, 12354S), ARID1B (Santa Cruz, sc-32762X), SMARCA4 (Abcam, ab110641) or H3K27ac (Abcam, ab4729) antibody respectively. The beads were washed three times with Wash Buffer (50 mM Hepes-KOH, pH 7.6, 500 mM LiCl, 1 mM EDTA, 1\% NP-40, 0.7\% Na-deoxycholate), and washed once with $1 \mathrm{~mL}$ TE buffer containing $50 \mathrm{mM} \mathrm{NaCl}$. The DNA was eluted with $210 \mu \mathrm{L}$ of Elution Buffer $(50 \mathrm{mM}$ Tris$\mathrm{HCl}, \mathrm{pH}$ 8.0, $10 \mathrm{mM}$ EDTA, 1\% SDS). The cross-links were reversed by incubated at $65^{\circ} \mathrm{C}$ overnight. $200 \mu \mathrm{L}$ of TE buffer was added to each tube, and the RNA was degraded by incubation with $16 \mu \mathrm{L}$ of $25 \mathrm{mg} / \mathrm{mL}$ RNase $A$ at $37^{\circ} \mathrm{C}$ for $60 \mathrm{~min}$. The protein was degraded by adding $4 \mu \mathrm{L}$ of $20 \mathrm{mg} / \mathrm{mL}$ proteinase $\mathrm{K}$ and incubating at $55^{\circ} \mathrm{C}$ for $60 \mathrm{~min}$. The DNA was then purified by phenol:chloroform:isoamyl alcohol extraction, and resuspended in $50 \mu \mathrm{L} \mathrm{ddH2O}$. The fragments of HERVH DNA were detected by qPCR (Supplementary Table 8). 
The RNAscope ${ }^{\mathrm{TM}}$ in situ hybridization (ISH)

The colon cancer tissue array (HCol-Ade180Sur) was purchased from Shanghai Biochip Co. Ltd (Shanghai, China). The RNAscope analysis with probes targeting the HERVH-gag sequence was performed using the RNAscope Multiplex Fluorescent Reagent Kit v2 (ACD bio, 323100) according to the manufacturer's protocol. The HERVH consensus sequence used for probe design was listed in Supplementary Table 9. Following the RNAscope staining, the tissue array was imaged with a LSM880 confocal microscope (Zeiss).

RNA-FISH combined with immunofluorescence

RNA-FISH combined with immunofluorescence was performed as previously described58. Cells cultured on poly-L-lysine-coated coverglasses were fixed with $10 \%$ formaldehyde in DPBS for $10 \mathrm{~min}$. After three washes in DPBS, cells were permeabilized with $0.5 \%$ Triton-X100 for $10 \mathrm{~min}$. The cells were then washed three times in DPBS and blocked with $4 \%$ Bovine Serum Albumin for $30 \mathrm{~min}$. The cells were incubated with the indicated primary antibody diluted in DPBS overnight, washed three times in DPBS, and incubated again with the secondary antibody for 1 hour. After washing twice with DPBS, the cells were fixed again with $10 \%$ formaldehyde in DPBS for $10 \mathrm{~min}$. Following two washes with DPBS, the cells were further washed in Wash Buffer I (20\% Stellaris RNA FISH Wash Buffer A (Biosearch Technologies, Inc., SMF-WA1-60), 10\% Deionized Formamide (Invitrogen, AM9342) in RNase-free water) for 5 min. The RNA probe (Stellaris) in hybridization buffer was added to the cells and incubated at $37^{\circ} \mathrm{C}$ for 16 hours. After washing with Wash Buffer I at $37^{\circ} \mathrm{C}$ for $30 \mathrm{~min}$, the cells were stained with $1 \mu \mathrm{g} / \mathrm{mL}$ DAPI for $5 \mathrm{~min}$. The cells were then washed with Wash Buffer B (Biosearch Technologies, Inc., SMF-WA1-60) for 5 min, and rinsed once in water before mounting with SlowFade Diamond Antifade Mountant (Invitrogen, S36963). The sequence of the RNA probe (Stellaris) was listed in Supplementary Table 10.

RNA-FISH and immunofluorescence with organoids

After dissolving the Matrigel with ice-cold cell recovery solution (Corning, 354253), the organoids were placed on a poly-L-lysine-coated glass slide for $30 \mathrm{~min}$. The organoids attached to the slide were fixed with $10 \%$ formaldehyde for $45 \mathrm{~min}$ at $4{ }^{\circ} \mathrm{C}$, and washed with DPBS for three times. The organoids were then permeabilized with $0.5 \%$ Triton-X100 for 15 min and washed with DPBS for two times. After one wash with Wash Buffer A for 5 min, the organoids were hybridized with the RNA-FISH probe overnight at $37^{\circ} \mathrm{C}$. After one wash with Wash Buffer A for $30 \mathrm{~min}$ at $37^{\circ} \mathrm{C}$, the organoids were stained with $1 \mu \mathrm{g} / \mathrm{mL}$ DAPI in Wash Buffer A for another 30 min, and washed twice with Wash Buffer B for 30 min. The organoids were rinsed with ddH2O and mounted with SlowFade Diamond Antifade Mountant (Invitrogen, S36963). The images were taken with a LSM880 confocal microscope (Zeiss). 
The immunofluorescence of organoids was performed as previously described 94 . The organoids cultured in 96-well plate were washed once with DPBS without disrupting the Matrigel, and then $200 \mu \mathrm{L}$ of ice-cold cell recovery solution (Corning, 354253) was added and incubated at $4{ }^{\circ} \mathrm{C}$ for 1 hour with shaking at 60 rpm. After the Matrigel was dissolved, the organoids were rinsed out using ice-cold PBS with $1 \%$ BSA and spun down at $70 \mathrm{~g}$ for $3 \mathrm{~min}$ at $4{ }^{\circ} \mathrm{C}$. The pellet of organoids was resuspended in $1 \mathrm{~mL}$ of $10 \%$ formaldehyde in DPBS, and incubated at $4{ }^{\circ} \mathrm{C}$ for $45 \mathrm{~min} .9 \mathrm{~mL}$ of ice-cold PBT (0.1\% Tween 20 in DPBS) was added and incubated at $4{ }^{\circ} \mathrm{C}$ for $10 \mathrm{~min}$. The organoids were then spun down at $70 \mathrm{~g}$ for $5 \mathrm{~min}$ at 4 ${ }^{\circ} \mathrm{C}$, resuspended in $200 \mu \mathrm{L}$ ice-cold OWB (0.1\% Triton X-100, 0.2\% BSA in DPBS), and transferred into 24well plate precoated with polyHEMA. Following incubation at $4{ }^{\circ} \mathrm{C}$ for $15 \mathrm{~min}, 200 \mu \mathrm{L}$ of the indicated primary antibody diluted in OWB was added and incubated overnight at $4^{\circ} \mathrm{C}$ with shaking at $60 \mathrm{rpm}$. The next day, $1 \mathrm{~mL}$ of OWB was added into each well. After all the organoids were settled at the bottom of the well, the OWB was removed with just $200 \mu \mathrm{L}$ left in each well. The organoids were washed three times with $1 \mathrm{~mL}$ of $O W B$ and incubated at $4{ }^{\circ} \mathrm{C}$ for 2 hours with shaking at $60 \mathrm{rpm}$. The OWB was removed with just $200 \mu \mathrm{L}$ left in each well, and then $200 \mu \mathrm{L}$ of secondary antibody diluted at 1:200 in OWB was added and incubated overnight at $4{ }^{\circ} \mathrm{C}$ with shaking at $60 \mathrm{rpm}$. After the incubation, the organoids were washed once with OWB, and $200 \mu \mathrm{L}$ of $2 \mu \mathrm{g} / \mathrm{mL}$ DAPI in OWB was added and incubated at $4{ }^{\circ} \mathrm{C}$ for $30 \mathrm{~min}$. The organoids were then washed two times with $\mathrm{OWB}$, transferred to 1.5-mL Eppendorf tube, and spun down at $70 \mathrm{~g}$ for $3 \mathrm{~min}$ at $4{ }^{\circ} \mathrm{C}$. The OWB was removed as much as possible without touching the organoids, and the organoids were resuspended with fructose-glycerol clearing solution $(60 \%$ glycerol and $2.5 \mathrm{M}$ fructose in $\mathrm{ddH} 2 \mathrm{O}$ ). Drew a $1 \times 2 \mathrm{~cm}$ rectangle in the middle of a slide, and placed 3 layers of sticky tape at both sides of the rectangle. The organoids were transferred into the middle of the rectangle, and put the coverslip on the top. The images were taken with a LSM880 confocal microscope (Zeiss).

\section{Fluorescence Recovery After Photobleaching (FRAP)}

The treated cells were plated into $35 \mathrm{~mm}$ glass bottom confocal dishes (NEST, 801001), and the FRAP experiment was performed on the Zeiss LSM880 Airyscan confocal microscope with a 63x PlanApochromat 1.4 NA oil objective. The Zeiss TempModule system was used to control the temperature (37 $\left.{ }^{\circ} \mathrm{C}\right)$, the humidity and the $\mathrm{CO} 2(5 \%)$ of the imaging system. After imaging for 3 frames, the cells were photo-bleached using $100 \%$ laser power with the $488 \mathrm{~nm}$ laser (iterations: 50, stop when intensity drops to $50 \%)$. The cells were then imaged again every two seconds. The images were analyzed and measured with ZEN 2 blue edition (Zeiss).

Code Availability.

All custom scripts are available from the authors upon request.

\section{References}


1. Feschotte, C. \& Gilbert, C. Endogenous viruses: insights into viral evolution and impact on host biology. Nat Rev Genet 13, 283-96 (2012).

2. Thompson, P.J., Macfarlan, T.S. \& Lorincz, M.C. Long Terminal Repeats: From Parasitic Elements to Building Blocks of the Transcriptional Regulatory Repertoire. Mol Cell 62, 766-76 (2016).

3. Chuong, E.B., Elde, N.C. \& Feschotte, C. Regulatory activities of transposable elements: from conflicts to benefits. Nat Rev Genet 18, 71-86 (2017).

4. Cosby, R.L., Chang, N.C. \& Feschotte, C. Host-transposon interactions: conflict, cooperation, and cooption. Genes Dev 33, 1098-1116 (2019).

5. Rebollo, R., Romanish, M.T. \& Mager, D.L. Transposable elements: an abundant and natural source of regulatory sequences for host genes. Annu Rev Genet 46, 21-42 (2012).

6. Etchegaray, E., Naville, M., Volff, J.N. \& Haftek-Terreau, Z. Transposable element-derived sequences in vertebrate development. Mob DNA 12, 1 (2021).

7. Piriyapongsa, J., Marino-Ramirez, L. \& Jordan, I.K. Origin and evolution of human microRNAs from transposable elements. Genetics 176, 1323-37 (2007).

8. Kelley, D. \& Rinn, J. Transposable elements reveal a stem cell-specific class of long noncoding RNAs. Genome Biol 13, R107 (2012).

9. Jacques, P.E., Jeyakani, J. \& Bourque, G. The majority of primate-specific regulatory sequences are derived from transposable elements. PLoS Genet 9, e1003504 (2013).

10. Kapusta, A. et al. Transposable elements are major contributors to the origin, diversification, and regulation of vertebrate long noncoding RNAs. PLoS Genet 9, e1003470 (2013).

11. Ito, J. et al. Systematic identification and characterization of regulatory elements derived from human endogenous retroviruses. PLoS Genet 13, e1006883 (2017).

12. Ramsay, L. et al. Conserved expression of transposon-derived non-coding transcripts in primate stem cells. BMC Genomics 18, 214 (2017).

13. Kunarso, G. et al. Transposable elements have rewired the core regulatory network of human embryonic stem cells. Nat Genet 42, 631-4 (2010).

14. Fort, A. et al. Deep transcriptome profiling of mammalian stem cells supports a regulatory role for retrotransposons in pluripotency maintenance. Nat Genet 46, 558-66 (2014).

15. Lu, X. et al. The retrovirus HERVH is a long noncoding RNA required for human embryonic stem cell identity. Nat Struct Mol Biol 21, 423-5 (2014).

16. Wang, J. et al. Primate-specific endogenous retrovirus-driven transcription defines naive-like stem cells. Nature 516, 405-9 (2014).

17. Friedli, M. \& Trono, D. The developmental control of transposable elements and the evolution of higher species. Annu Rev Cell Dev Bio/ 31, 429-51 (2015).

18. Garcia-Perez, J.L., Widmann, T.J. \& Adams, I.R. The impact of transposable elements on mammalian development. Development 143, 4101-4114 (2016). 
19. Romer, C., Singh, M., Hurst, L.D. \& Izsvak, Z. How to tame an endogenous retrovirus: HERVH and the evolution of human pluripotency. Curr Opin Virol 25, 49-58 (2017).

20. Rodriguez-Terrones, D. \& Torres-Padilla, M.E. Nimble and Ready to Mingle: Transposon Outbursts of Early Development. Trends Genet 34, 806-820 (2018).

21. Pontis, J. et al. Hominoid-Specific Transposable Elements and KZFPs Facilitate Human Embryonic Genome Activation and Control Transcription in Naive Human ESCs. Cell Stem Cell 24, 724-735 e5 (2019).

22. Zhang, Y. et al. Transcriptionally active HERV-H retrotransposons demarcate topologically associating domains in human pluripotent stem cells. Nat Genet 51, 1380-1388 (2019).

23. Lu, J.Y. et al. Homotypic clustering of L1 and B1/Alu repeats compartmentalizes the 3D genome. Cell Res (2021).

24. Weiss, R.A. The discovery of endogenous retroviruses. Retrovirology 3, 67 (2006).

25. Jern, P., Sperber, G.O. \& Blomberg, J. Definition and variation of human endogenous retrovirus $H$. Virology 327, 93-110 (2004).

26. Izsvak, Z., Wang, J., Singh, M., Mager, D.L. \& Hurst, L.D. Pluripotency and the endogenous retrovirus HERVH: Conflict or serendipity? Bioessays 38, 109 - 17 (2016).

27. Gerdes, P., Richardson, S.R., Mager, D.L. \& Faulkner, G.J. Transposable elements in the mammalian embryo: pioneers surviving through stealth and service. Genome Biol 17, 100 (2016).

28. Groh, S. \& Schotta, G. Silencing of endogenous retroviruses by heterochromatin. Cell Mol Life Sci 74, 2055-2065 (2017).

29. Liu, J. et al. The RNA m(6)A reader YTHDC1 silences retrotransposons and guards ES cell identity. Nature 591, 322-326 (2021).

30. Xu, W. et al. METTL3 regulates heterochromatin in mouse embryonic stem cells. Nature 591, 317321 (2021).

31. Li, P. et al. Rif1 promotes a repressive chromatin state to safeguard against endogenous retrovirus activation. Nucleic Acids Res 45, 12723-12738 (2017).

32. He, J. et al. Transposable elements are regulated by context-specific patterns of chromatin marks in mouse embryonic stem cells. Nat Commun 10, 34 (2019).

33. Goke, J. et al. Dynamic transcription of distinct classes of endogenous retroviral elements marks specific populations of early human embryonic cells. Cell Stem Cell 16, 135-41 (2015).

34. Yi, J.M., Kim, H.M. \& Kim, H.S. Human endogenous retrovirus HERV-H family in human tissues and cancer cells: expression, identification, and phylogeny. Cancer Lett 231, 228-39 (2006).

35. Wentzensen, N. et al. Expression of an endogenous retroviral sequence from the HERV-H group in gastrointestinal cancers. Int J Cancer 121, 1417-23 (2007).

36. Perot, P. et al. Expression of young HERV-H loci in the course of colorectal carcinoma and correlation with molecular subtypes. Oncotarget 6, 40095-111 (2015). 
37. Mullins, C.S. et al. Generation, Characterization and Application of Antibodies Directed against HERVH Gag Protein in Colorectal Samples. PLoS One 11, e0153349 (2016).

38. Lynch-Sutherland, C.F., Chatterjee, A., Stockwell, P.A., Eccles, M.R. \& Macaulay, E.C. Reawakening the Developmental Origins of Cancer Through Transposable Elements. Front Oncol 10, 468 (2020).

39. Jansz, N. \& Faulkner, G.J. Endogenous retroviruses in the origins and treatment of cancer. Genome Biol 22, 147 (2021).

40. Wang, Y., Hoang, L., Ji, J.X. \& Huntsman, D.G. SWI/SNF Complex Mutations in Gynecologic Cancers: Molecular Mechanisms and Models. Annu Rev Pathol 15, 467-492 (2020).

41. Lu, C. \& Allis, C.D. SWI/SNF complex in cancer. Nat Genet 49, 178-179 (2017).

42. Mathur, R. et al. ARID1A loss impairs enhancer-mediated gene regulation and drives colon cancer in mice. Nat Genet 49, 296-302 (2017).

43. Arnaud, O., Le Loarer, F. \& Tirode, F. BAFfling pathologies: Alterations of BAF complexes in cancer. Cancer Lett 419, 266-279 (2018).

44. Luo, Q., Wu, X. \& Liu, Z. Remodeling of the ARID1A tumor suppressor. Cancer Lett 491, 1-10 (2020).

45. Berns, K. et al. ARID1A mutation sensitizes most ovarian clear cell carcinomas to BET inhibitors. Oncogene 37, 4611-4625 (2018).

46. Nagarajan, S. et al. ARID1A influences HDAC1/BRD4 activity, intrinsic proliferative capacity and breast cancer treatment response. Nat Genet 52, 187-197 (2020).

47. Wilson, M.R. et al. ARID1A Mutations Promote P300-Dependent Endometrial Invasion through SuperEnhancer Hyperacetylation. Cell Rep 33, 108366 (2020).

48. Cancer Genome Atlas, N. Comprehensive molecular characterization of human colon and rectal cancer. Nature 487, 330-7 (2012).

49. Ghandi, M. et al. Next-generation characterization of the Cancer Cell Line Encyclopedia. Nature 569, 503-508 (2019).

50. Kelso, T.W.R. et al. Chromatin accessibility underlies synthetic lethality of SWI/SNF subunits in ARID1A-mutant cancers. Elife 6(2017).

51. Wang, X. et al. Two related ARID family proteins are alternative subunits of human SWI/SNF complexes. Biochem J 383, 319-25 (2004).

52. Nagl, N.G., Jr., Wang, X., Patsialou, A., Van Scoy, M. \& Moran, E. Distinct mammalian SWI/SNF chromatin remodeling complexes with opposing roles in cell-cycle control. EMBO J 26, 752-63 (2007).

53. He, S. et al. Structure of nucleosome-bound human BAF complex. Science 367, 875-881 (2020).

54. Helming, K.C. et al. ARID1B is a specific vulnerability in ARID1A-mutant cancers. Nat Med 20, 251-4 (2014).

55. Trizzino, M. et al. The Tumor Suppressor ARID1A Controls Global Transcription via Pausing of RNA Polymerase II. Cell Rep 23, 3933-3945 (2018). 
56. Santoni, F.A., Guerra, J. \& Luban, J. HERV-H RNA is abundant in human embryonic stem cells and a precise marker for pluripotency. Retrovirology 9, 111 (2012).

57. Lewis, M.W., Li, S. \& Franco, H.L. Transcriptional control by enhancers and enhancer RNAs. Transcription-Austin 10, 171-186 (2019).

58. Sabari, B.R. et al. Coactivator condensation at super-enhancers links phase separation and gene control. Science 361(2018).

59. Gibson, B.A. et al. Organization of Chromatin by Intrinsic and Regulated Phase Separation. Cell 179, 470-484 e21 (2019).

60. Yin, M. et al. Potent BRD4 inhibitor suppresses cancer cell-macrophage interaction. Nat Commun 11, 1833 (2020).

61. Bitler, B.G. et al. Synthetic lethality by targeting EZH2 methyltransferase activity in ARID1A-mutated cancers. Nat Med 21, 231-8 (2015).

62. Kim, K.H. et al. SWI/SNF-mutant cancers depend on catalytic and non-catalytic activity of EZH2. Nat Med 21, 1491-6 (2015).

63. Bitler, B.G. et al. ARID1A-mutated ovarian cancers depend on HDAC6 activity. Nat Cell Bio/ 19, 962973 (2017).

64. Fukumoto, T. et al. Repurposing Pan-HDAC Inhibitors for ARID1A-Mutated Ovarian Cancer. Cell Rep 22, 3393-3400 (2018).

65. Wu, C. et al. Targeting AURKA-CDC25C axis to induce synthetic lethality in ARID1A-deficient colorectal cancer cells. Nat Commun 9, 3212 (2018).

66. Ogiwara, H. et al. Targeting the Vulnerability of Glutathione Metabolism in ARID1A-Deficient Cancers. Cancer Cel/ 35, 177-190 e8 (2019).

67. Sun, X. et al. Suppression of the SWI/SNF Component Arid1 a Promotes Mammalian Regeneration. Cell Stem Cell 18, 456-66 (2016).

68. Hiramatsu, Y. et al. Arid1a is essential for intestinal stem cells through Sox9 regulation. Proc Natl Acad Sci U S A 116, 1704-1713 (2019).

69. Wu, S. et al. ARID1A spatially partitions interphase chromosomes. Sci Adv 5, eaaw5294 (2019).

70. Yuan, K. \& O'Farrell, P.H. TALE-light imaging reveals maternally guided, H3K9me2/3-independent emergence of functional heterochromatin in Drosophila embryos. Genes Dev 30, 579-93 (2016).

71. Molaro, A. \& Malik, H.S. Hide and seek: how chromatin-based pathways silence retroelements in the mammalian germline. Curr Opin Genet Dev 37, 51-58 (2016).

72. Bourque, G. et al. Ten things you should know about transposable elements. Genome Bio/ 19, 199 (2018).

73. Grow, E.J. et al. Intrinsic retroviral reactivation in human preimplantation embryos and pluripotent cells. Nature 522, 221-5 (2015).

74. Downey, R.F. et al. Human endogenous retrovirus $\mathrm{K}$ and cancer: Innocent bystander or tumorigenic accomplice? Int J Cancer 137, 1249-57 (2015). 
75. Jin, X. et al. The endogenous retrovirus-derived long noncoding RNA TROJAN promotes triplenegative breast cancer progression via ZMYND8 degradation. Sci Adv 5, eaat9820 (2019).

76. Curty, G. et al. Human Endogenous Retrovirus K in Cancer: A Potential Biomarker and Immunotherapeutic Target. Viruses 12(2020).

77. Deniz, O. et al. Endogenous retroviruses are a source of enhancers with oncogenic potential in acute myeloid leukaemia. Nat Commun 11, 3506 (2020).

78. Babaian, A. \& Mager, D.L. Endogenous retroviral promoter exaptation in human cancer. Mob DNA 7, 24 (2016).

79. Jang, H.S. et al. Transposable elements drive widespread expression of oncogenes in human cancers. Nat Genet 51, 611-617 (2019).

80. Liu, J. et al. An Integrated TCGA Pan-Cancer Clinical Data Resource to Drive High-Quality Survival Outcome Analytics. Cell 173, 400-416 e11 (2018).

81. Kim, S.K. et al. A nineteen gene-based risk score classifier predicts prognosis of colorectal cancer patients. Mol Oncol 8, 1653-66 (2014).

82. Dobin, A. et al. STAR: ultrafast universal RNA-seq aligner. Bioinformatics 29, 15-21 (2013).

83. Liao, Y., Smyth, G.K. \& Shi, W. featureCounts: an efficient general purpose program for assigning sequence reads to genomic features. Bioinformatics 30, 923-30 (2014).

84. Love, M.I., Huber, W. \& Anders, S. Moderated estimation of fold change and dispersion for RNA-seq data with DESeq2. Genome Bio/ 15, 550 (2014).

85. Yu, G., Wang, L.G., Han, Y. \& He, Q.Y. clusterProfiler: an R package for comparing biological themes among gene clusters. OMICS 16, 284-7 (2012).

86. Langmead, B. \& Salzberg, S.L. Fast gapped-read alignment with Bowtie 2. Nat Methods 9, 357-9 (2012).

87. Danecek, P. et al. Twelve years of SAMtools and BCFtools. Gigascience 10(2021).

88. Zhang, Y. et al. Model-based analysis of ChIP-Seq (MACS). Genome Biol 9, R137 (2008).

89. Quinlan, A.R. \& Hall, I.M. BEDTools: a flexible suite of utilities for comparing genomic features. Bioinformatics 26, 841-2 (2010).

90. Ramirez, F. et al. deepTools2: a next generation web server for deep-sequencing data analysis. Nucleic Acids Res 44, W160-5 (2016).

91. Robinson, J.T. et al. Integrative genomics viewer. Nat Biotechnol 29, 24-6 (2011).

92. Driehuis, E., Kretzschmar, K. \& Clevers, H. Establishment of patient-derived cancer organoids for drugscreening applications. Nat Protoc 15, 3380-3409 (2020).

93. Liu, T. et al. MYC predetermines the sensitivity of gastrointestinal cancer to antifolate drugs through regulating TYMS transcription. EBioMedicine 48, 289-300 (2019).

94. Dekkers, J.F. et al. High-resolution 3D imaging of fixed and cleared organoids. Nat Protoc 14, 17561771 (2019). 


\section{Declarations}

\section{Acknowledgements}

We gratefully acknowledge Prof. François Mallet for HERVH antibodies. We thank Dr. Kai Fu and Dr. Joong Sup Shim for cell lines, and colleagues in the center of medical genetics in Central South University and members of the Yuan lab for helpful discussion. This project has been supported by Central South University (2018CX032 to K.Y, $2019 z z t s 339$ to X.L, and the innovation-driven team project 2020CX016), Department of Science \& Technology of Hunan Province (grants 2017RS3013, 2017XK2011, 2018DK2015, and 2019SK1012 to K.Y, 2019JJ40478 to P.L, and the innovative team program 2019RS1010), the National Natural Science Foundation of China (grants 31771589 and 91853108 to K.Y, 81801426 to L.S). K.Y. is supported by the National Thousand Talents Program for Young Outstanding Scientists.

\section{Author contributions}

C.Y., X.L., X.H., P.L., and K.Y. conceived the project. X.H. initiated this project. C.Y., X.L., F.C., S.M., L.L., H.L., X.H., R.W., L.S., N.Z., Y.M., Y.S., and P.L. performed the experiments. X.L. and S.M. performed all the bioinformatic and statistical analyses with the supervisions from K.Y. and P.L. C.L. assisted with patient selection. S.H., H.S., and Z.Z. provided insightful suggestions in project design and manuscript writing. K.Y. wrote the manuscript with helps from C.Y. and X.L.

\section{Figures}


A

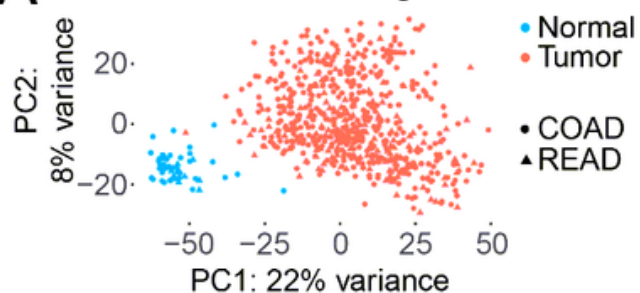

C

Differentially Expressed Repeats

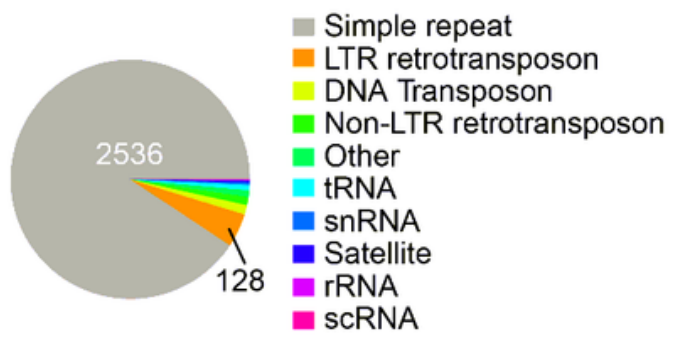

$E$

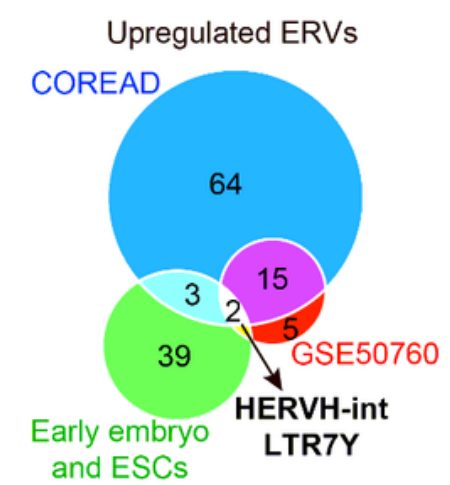

\section{B PCA based on repeats}
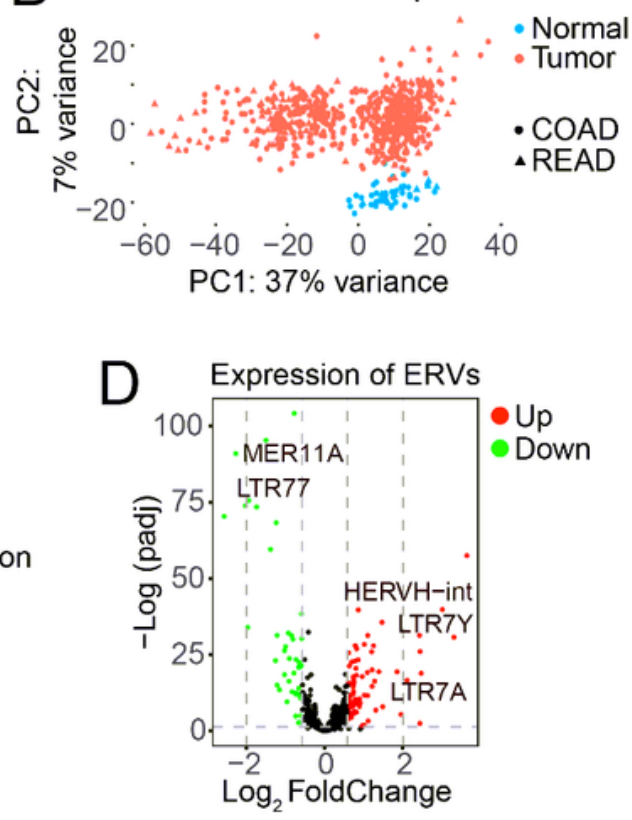
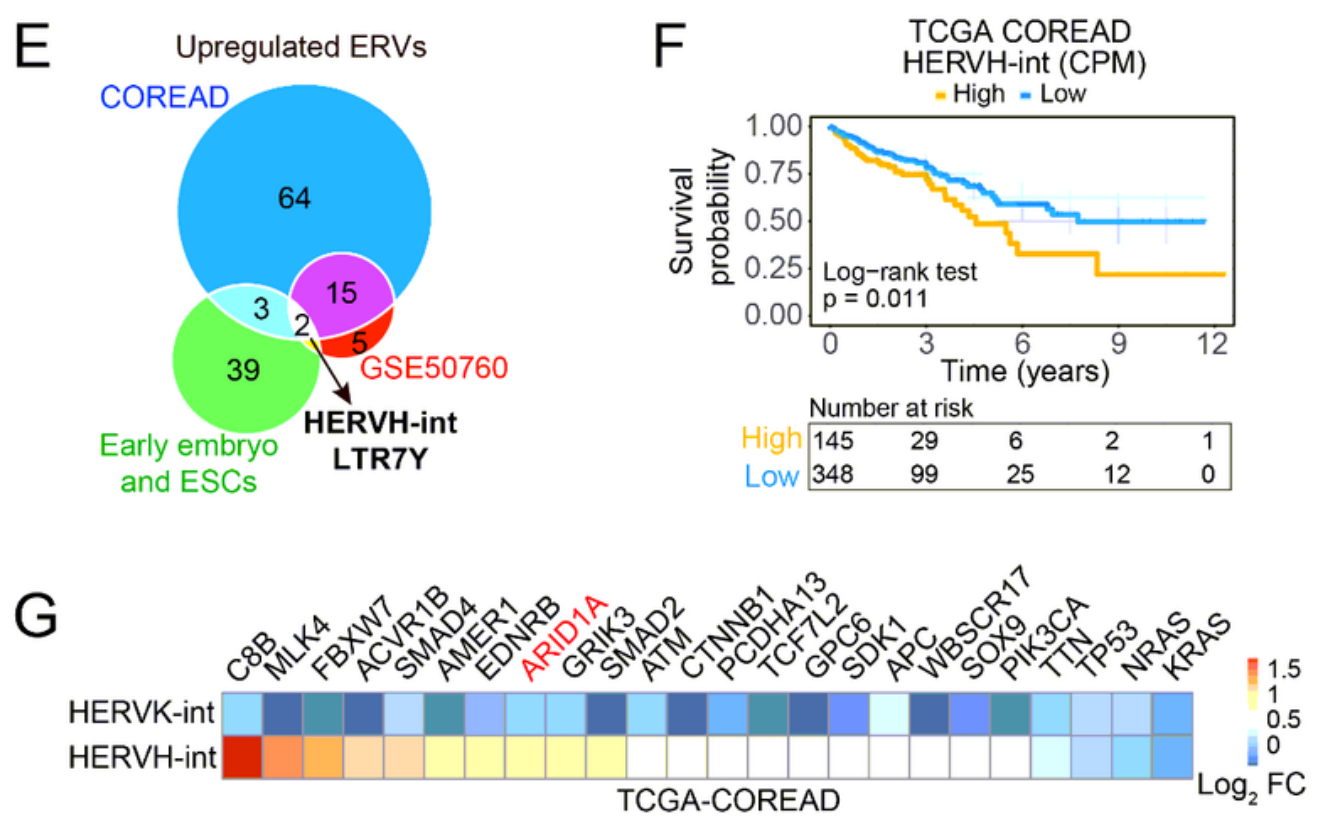

$\mathrm{H}$

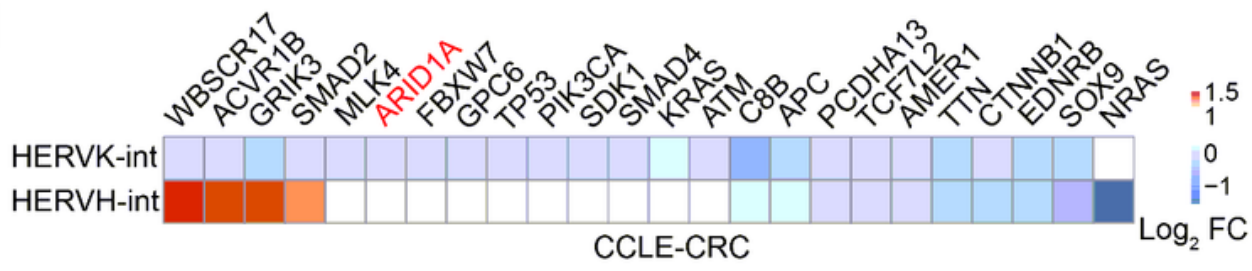

\section{Figure 1}

Characterization of HERVH expression in CRCs. (A) Principal component analysis (PCA) of gene expression of 51 normal and 631 CRC tumor tissues from the TCGA COREAD dataset. (B) PCA based on the expression of repetitive sequences in the same TCGA dataset. (C) Classification of differentially expressed repetitive sequences (adjusted p-value $<0.05$ and |Log2 FoldChangel $>0.585$ ). (D) Volcano plot of differentially expressed ERVs. Up (red) and down (green) regulated ERVs are determined with the 
cut-off values of adjusted p-value $<0.05$ and |Log2 FoldChange| $>0.585$. (E) Overlap analysis of upregulated ERVs in CRCs samples and early embryonic cells identifies the internal coding sequences of HERVH (HERVH-int) and its corresponding LTR (LTR7Y) as the commonly upregulated elements. (F) Survival analysis based on the expression level of HERVH-int and the overall survival (OS) from 493 patients with AJCC pathologic tumor stage greater than I. The mean expression value of HERVH-int is used to demarcate the HERVH-int-High (145 patients) and HERVH-int-Low (348 patients) groups. (G) Correlation analysis of HERVH-int expression and mutational status of the most frequently mutated genes in CRCs using the TCGA dataset. $(\mathrm{H})$ Correlation of HERVH-int expression and gene mutations in $\mathrm{CRC}$ cell lines from the CCLE dataset. 


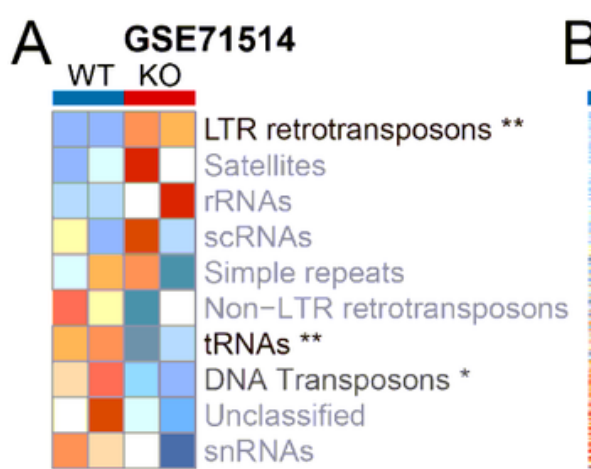

All repeats

$\mathrm{D}$

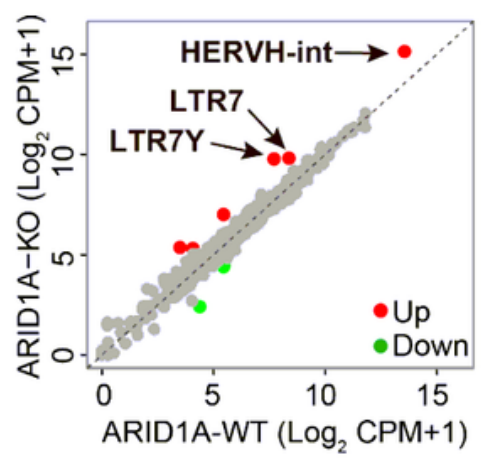

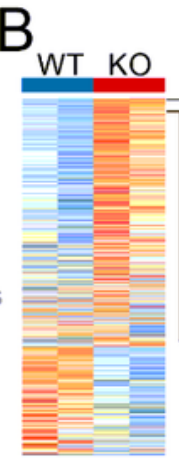

All ERVs

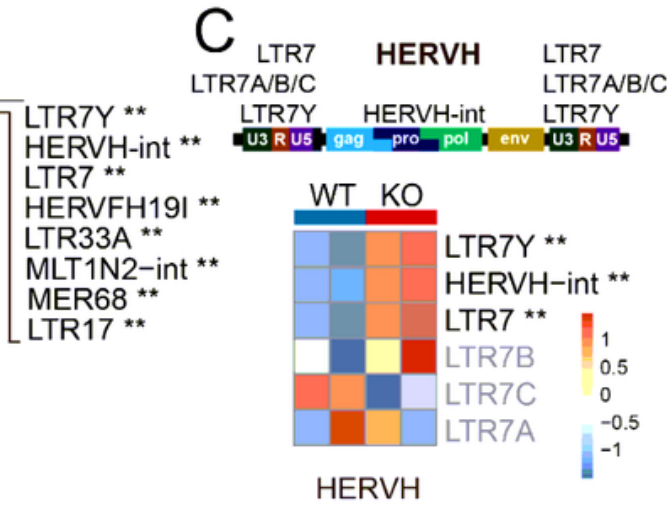

E GSE71514

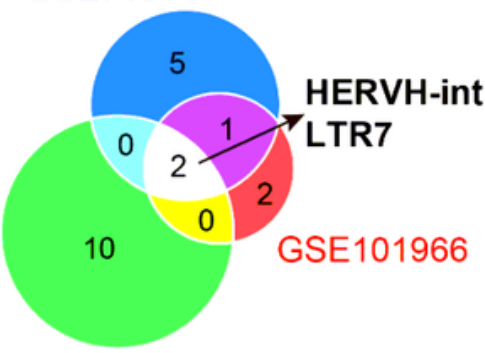

This study

$\mathrm{F}$
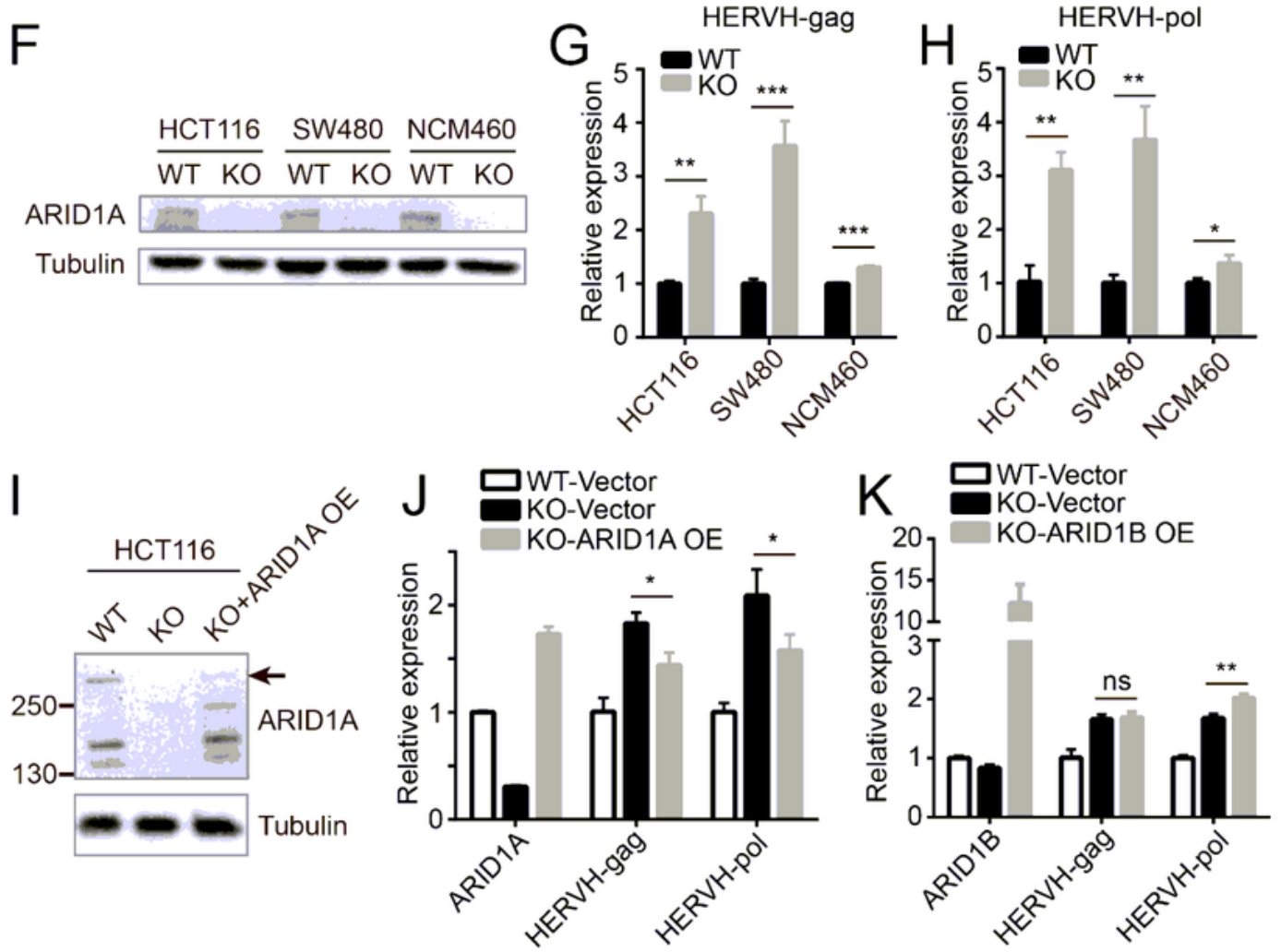

Figure 2

ARID1A loss leads to upregulation of HERVH. (A-C) Heatmaps of the expression of different repetitive sequences in wild type (WT) and ARID1A knockout (KO) HCT116 cells. The differential expression is tested based on a model using the negative binomial distribution (adjusted $p$ values are labeled as *padj $<0.05, * *$ padj $<0.01$ ). The schematic of a typical full-length HERVH element is shown in (C). (D) A scatter plot of the expression of all 580 ERVs in WT and KO HCT116 cells. The up- or downregulated ERVs are 
labeled in red or green respectively (|Log2 FoldChangel $>1$ and adjusted $p$-value $<0.05$ ). (E) Venn diagram showing that HERVH is repetitively upregulated in three independent sequencing experiments with HCT116 ARID1A WT and KO cells. (F) Western blots of different ARID1A WT and KO cell lines. (G-H) qPCR analyses of HERVH expression in ARID1A WT and KO cell lines using two different primer sets. * $p<$ $0.05,{ }^{*} \mathrm{p}<0.01,{ }^{* \star} \mathrm{p}<0.001$ by t-test. (I) Western blots showing ARID1A protein levels in WT, KO, and ARID1A rescued KO HCT116 cells. Arrow indicates the full-length ARID1A band. $(\mathrm{J})$ qPCR analysis of ARID1A and HERVH expression in the indicated groups of cells. ${ }^{*} p<0.05$ by t-test. (K) qPCR analysis of ARID1B and HERVH expression. ${ }^{*} \mathrm{p} p<0.01$ by t-test. 


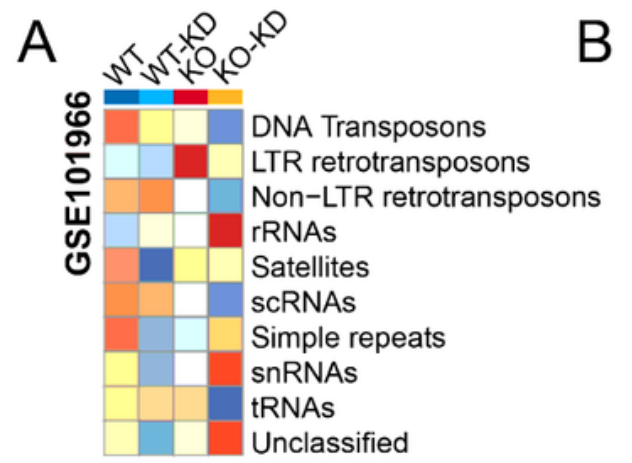

D

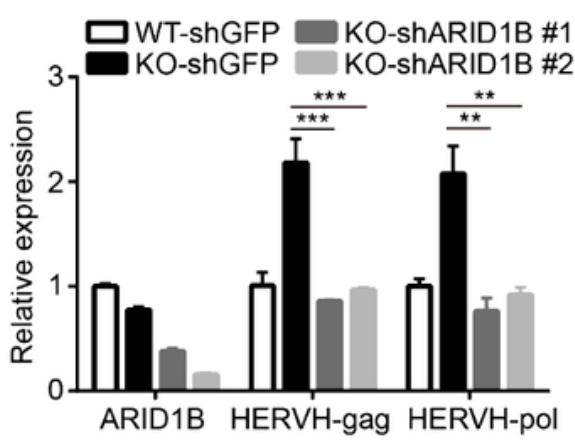

$\mathrm{F}$

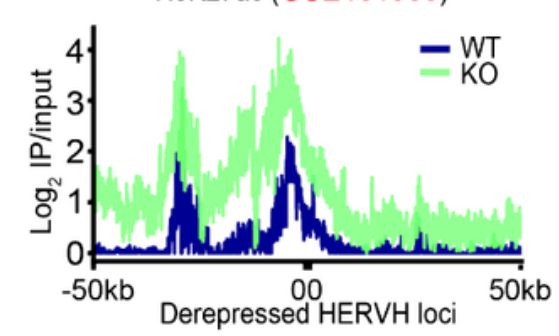

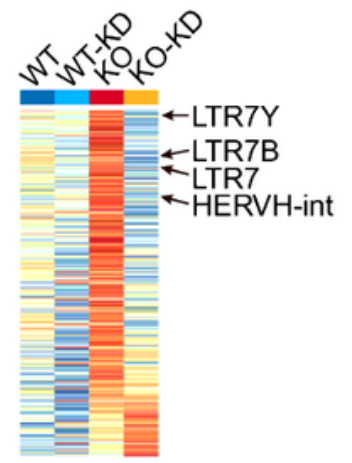

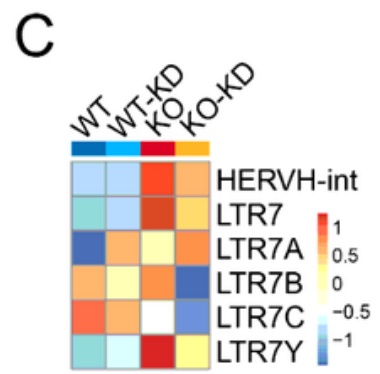

$\mathrm{E}$

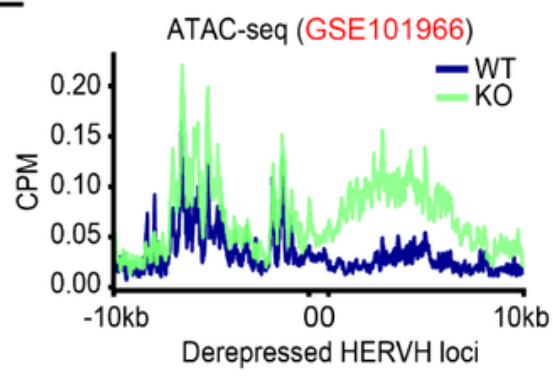

G

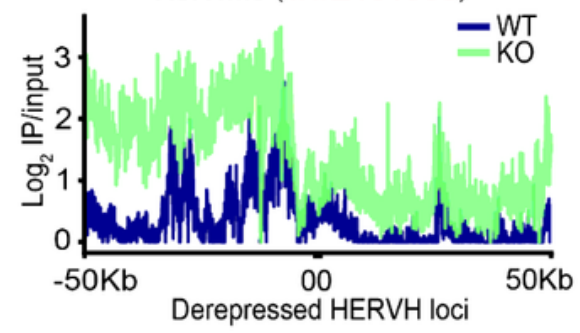

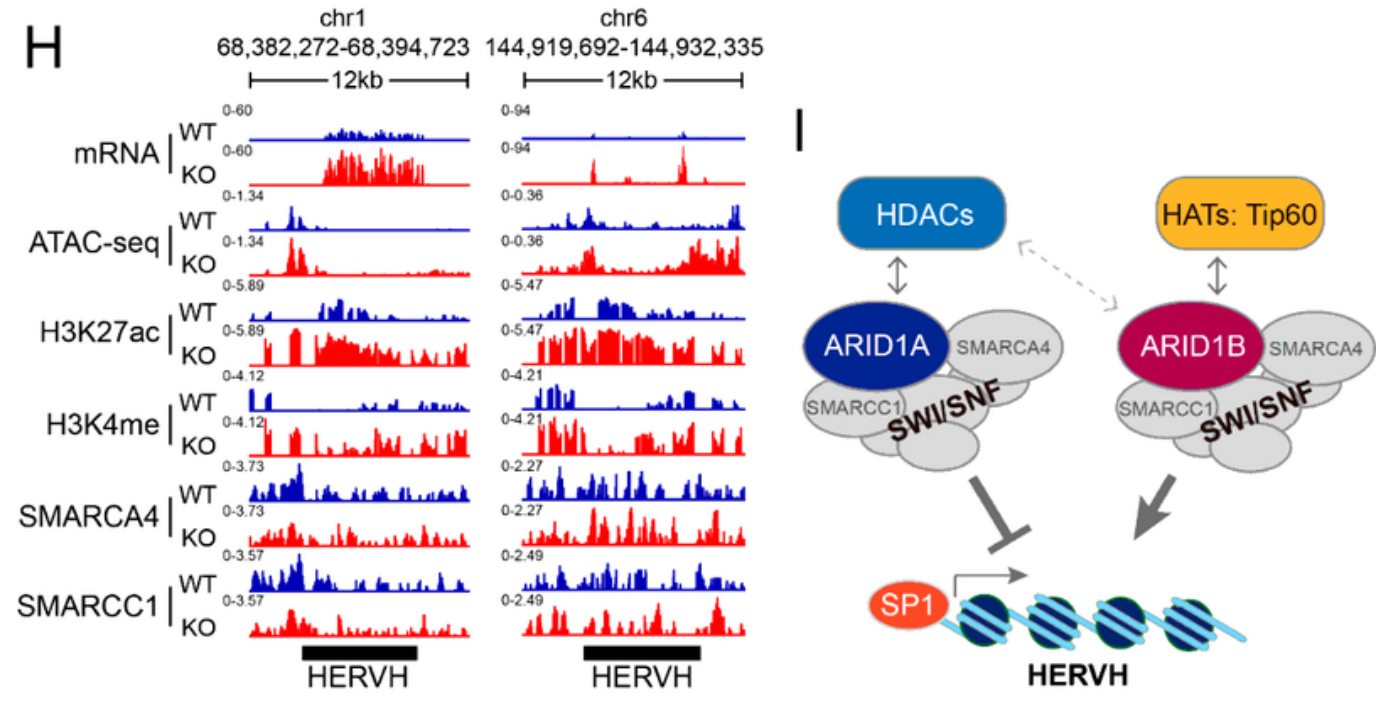

Figure 3

ARID1B is required for the upregulation of HERVH in the absence of ARID1A. (A-C) Heatmaps of the expression of different repetitive sequences in WT or ARID1A KO HCT116 cells treated with scrambled control or ARID1B (-KD) shRNAs. (D) qPCR results showing the expression levels of ARID1B and HERVH in ARID1A WT and KO cells treated with shRNA targeting GFP or ARID1B. ${ }^{\star \star} p<0.01,{ }^{\star \star \star} p<0.001$ by t-test. (E) ATAC-seq results from ARID1A WT and KO HCT116 cells (GSE101966) demonstrate increased 
chromatin accessibility at the derepressed HERVH loci in ARID1A KO cells. (F-G) ChIP-seq data (GSE101966) show increased H3K27ac and H3K4me at the derepressed HERVH loci in ARID1A KO HCT116 cells. (H) Genomic snapshots of RNA-seq, ATAC-seq, and ChIP-seq signals at two representative HERVH loci. (I) Model showing the mutually exclusive relationship between ARID1A- and ARID1Bcontaining BAF complexes, their differential associations with HDACs and HATs, and the different regulatory functions imposed on HERVH loci.
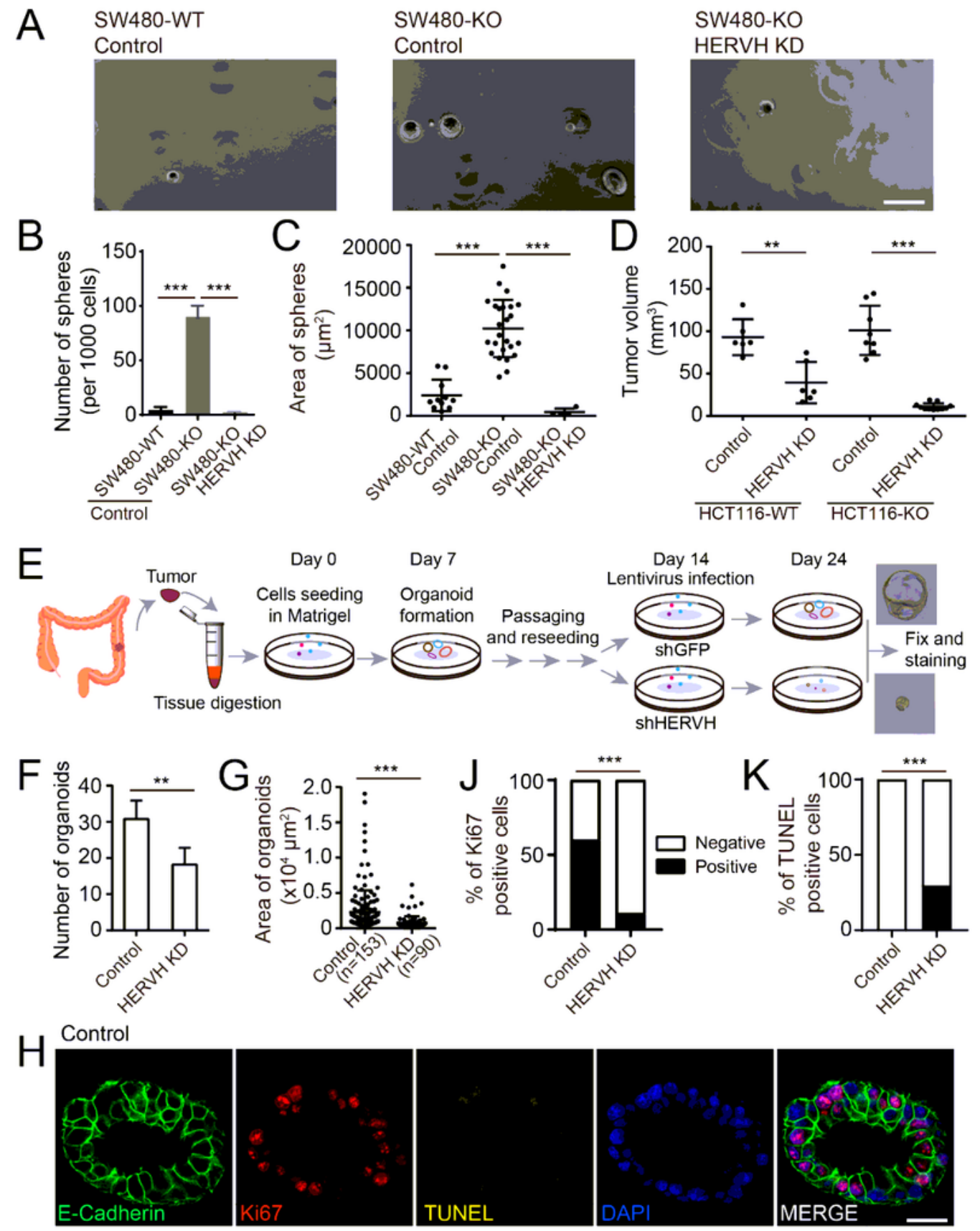

I HERVH KD
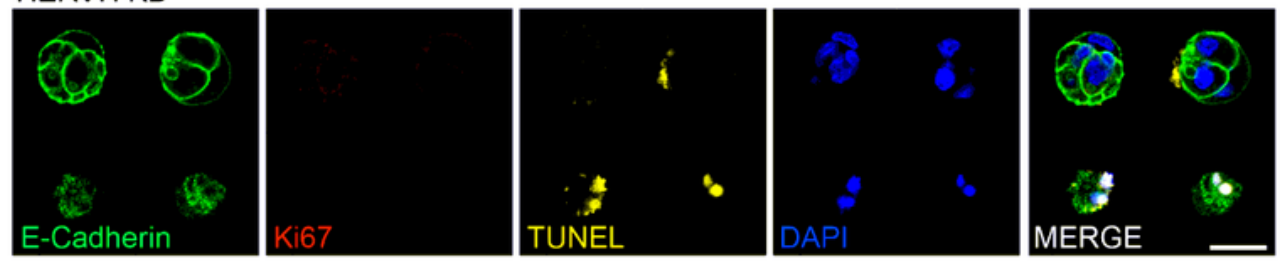

Figure 4 
HERVH is essential for the proliferation of CRC cells. (A) Representative brightfield images showing that knockdown of HERVH inhibits sphere formation of ARID1A KO SW480 cells. Bar: $130 \square \mathrm{m}$. (B-C) Quantifications of sphere number and sphere size of the indicated cells. ${ }^{* \star} \mathrm{p}<0.001$ by t-test. (D) HERVH knockdown suppresses tumor growth of WT and ARID1A KO HCT116 cells in mouse subcutaneous xenograft tumor models. ${ }^{\star \star} p<0.01,{ }^{\star \star \star} p<0.001$ by t-test. (E) Schematic illustrating the establishment and subsequent treatments of patient-derived CRC organoids. (F-G) HERVH knockdown decreases both the number and the size of CRC organoids. ${ }^{* \star} \mathrm{p}<0.01,{ }^{\star \star \star} \mathrm{p}<0.001$ by $\mathrm{t}$-test. $(\mathrm{H}-\mathrm{I})$ Representative images of control and HERVH shRNA treated organoids stained with E-Cadherin (green), Ki67 (red), TUNEL (yellow), and DAPI (blue). Bars: $34 \square \mathrm{m}$. (J-K) Percentages of Ki67 or TUNEL positive cells in control and HERVH shRNA treated CRC organoids. ${ }^{\star \star *} \mathrm{p}<0.001$ by chi-squared test. 


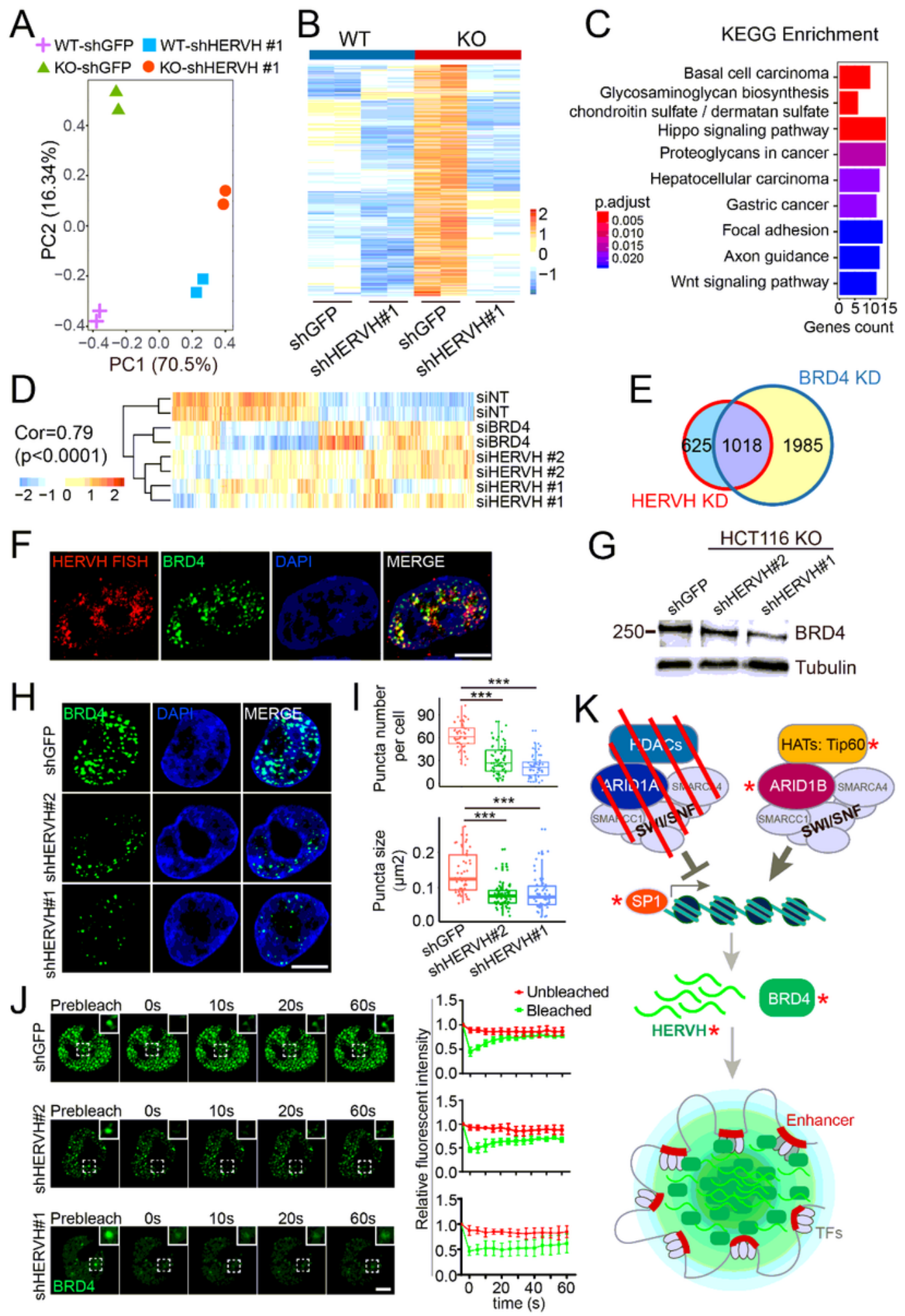

Figure 5

HERVH contributes to the formation of BRD4 puncta and its function in transcriptional regulation. (A) PCA analysis showing the impacts of HERVH knockdown on the global transcriptome of HCT116 WT and KO cells. (B) Heatmap highlighting a set of genes whose expression increase upon ARID1A loss but decrease again when HERVH is knocked down. (C) KEGG enrichment analysis of the HERVH-dependent genes. (D) Heatmap showing strong correlation of changes in transcriptome between knockdowns of 
HERVH and BRD4 in ARID1A KO HCT116 cells. (E) Venn diagram showing that 1018 genes are coregulated by BRD4 and HERVH. (F) Partial colocalization between HERVH FISH signals and GFP-BRD4 nuclear foci. Bar: $5 \square \mathrm{m}$. (G) Western blot showing decreased BRD4 protein level upon knockdown of HERVH in HCT116 ARID1A KO cells. (H) Representative images of BRD4 nuclear foci in control and HERVH shRNA treated cells. Bar: $5 \square \mathrm{m}$. (I) Quantifications of the number and size of BRD4 foci. ***p < 0.001 by Wilcox test. $(\mathrm{J})$ Fluorescence recovery after photo bleaching (FRAP) analysis of BRD4 foci after control or HERVH knockdown. Bar: $3.3 \square \mathrm{m}$. (K) A model summarizing how ARID1A loss upregulates HERVH and hence stimulates BRD4 nuclear foci formation and BRD4-mediated transcription. Red asterisks label potential targets of intervention.

\section{Supplementary Files}

This is a list of supplementary files associated with this preprint. Click to download.

- SupplementaryTable1.DerepressionofTEsinCRC.xlsx

- SupplementaryTable2.ARID1AlossactivatesHERVH.xIsx

- SupplementaryTable3.Arid1alossactivatesERVsubfamiliesinmouse.xlsx

- SupplementaryTable4.ARID1BactivatesHERVHintheabsenceofARID1A.xIsx

- SupplementaryTable5.H3K27acincreasesinactiveHERVHlocusafterKO.xlsx

- SupplementaryTable6.KDSP1decreasesHERVH.xIsx

- SupplementaryTable7.ARID1AlossactiavtesHERVHandBRD4coregulatedgenes.xlsx

- SupplementaryTable8.Oligonucleotidesequences.xlsx

- SupplementaryTable9.HERVHconsensussequenceforRNAscopeprobedesign.xlsx

- SupplementaryTable10.HERVHStellarisFISHProbes.xIsx

- SupplementaryTable11.Antibodies.xIsx

- Onlinefloatimage2.png

- Onlinefloatimage4.png

- Onlinefloatimage6.png

- Onlinefloatimage8.png

- Onlinefloatimage10.png 